\title{
POINT SEARCHING IN REAL SINGULAR COMPLETE INTERSECTION VARIETIES: ALGORITHMS OF INTRINSIC COMPLEXITY
}

\author{
BERND BANK, MARC GIUSTI, AND JOOS HEINTZ
}

Abstract. Let $X_{1}, \ldots, X_{n}$ be indeterminates over $\mathbb{Q}$ and let $X:=\left(X_{1}, \ldots\right.$, $\left.X_{n}\right)$. Let $F_{1}, \ldots, F_{p}$ be a regular sequence of polynomials in $\mathbb{Q}[X]$ of degree at most $d$ such that for each $1 \leq k \leq p$ the ideal $\left(F_{1}, \ldots, F_{k}\right)$ is radical. Suppose that the variables $X_{1}, \ldots, X_{n}$ are in generic position with respect to $F_{1}, \ldots, F_{p}$. Further, suppose that the polynomials are given by an essentially division-free circuit $\beta$ in $\mathbb{Q}[X]$ of size $L$ and non-scalar depth $\ell$.

We present a family of algorithms $\Pi_{i}$ and invariants $\delta_{i}$ of $F_{1}, \ldots, F_{p}, 1 \leq$ $i \leq n-p$, such that $\Pi_{i}$ produces on input $\beta$ a smooth algebraic sample point for each connected component of $\left\{x \in \mathbb{R}^{n} \mid F_{1}(x)=\cdots=F_{p}(x)=0\right\}$ where the Jacobian of $F_{1}=0, \ldots, F_{p}=0$ has generically rank $p$.

The sequential complexity of $\Pi_{i}$ is of order $L(n d)^{O(1)}\left(\min \left\{(n d)^{c n}, \delta_{i}\right\}\right)^{2}$ and its non-scalar parallel complexity is of order $O\left(n(\ell+\log n d) \log \delta_{i}\right)$. Here $c>0$ is a suitable universal constant. Thus, the complexity of $\Pi_{i}$ meets the already known worst case bounds. The particular feature of $\Pi_{i}$ is its pseudo-polynomial and intrinsic complexity character and this entails the best runtime behavior one can hope for. The algorithm $\Pi_{i}$ works in the non-uniform deterministic as well as in the uniform probabilistic complexity model. We also exhibit a worst case estimate of order $\left(n^{n} d\right)^{O(n)}$ for the invariant $\delta_{i}$. The reader may notice that this bound overestimates the extrinsic complexity of $\Pi_{i}$, which is bounded by $(n d)^{O(n)}$.

\section{INTRODUCTION}

Before we begin to explain the main results of this article and their motivations, we introduce some basic notions and notation.

Let $\mathbb{Q}, \mathbb{R}$ and $\mathbb{C}$ be the fields of rational, real and complex numbers, respectively, let $X:=\left(X_{1}, \ldots, X_{n}\right)$ be a vector of indeterminates over $\mathbb{C}$ and let $F_{1}, \ldots, F_{p}$ be a regular sequence of polynomials in $\mathbb{Q}[X]$ defining a closed, $\mathbb{Q}$-definable subvariety $S$ of the $n$-dimensional complex affine space $\mathbb{A}^{n}:=\mathbb{C}^{n}$. Thus $S$ is a non-empty equidimensional affine variety of dimension $n-p$, i.e., each irreducible component of $S$ is of dimension $n-p$. Said otherwise, $S$ is a closed subvariety of $\mathbb{A}^{n}$ of pure codimension $p$ (in $\left.\mathbb{A}^{n}\right)$.

Let $\mathbb{A}_{\mathbb{R}}^{n}:=\mathbb{R}^{n}$ be the $n$-dimensional real affine space. We denote by $S_{\mathbb{R}}:=$ $S \cap \mathbb{A}_{\mathbb{R}}^{n}$ the real trace of the complex variety $S$. Moreover, we denote by $\mathbb{P}^{n}$ the

Received by the editor August 16, 2011 and, in revised form, July 12, 2012.

2010 Mathematics Subject Classification. Primary 68W30 14B05, 14P05, 14B07, 68W10.

Key words and phrases. Real polynomial equation solving, intrinsic complexity, singularities, polar, copolar and bipolar varieties, degree of variety.

This research was partially supported by the following Argentinian, French and Spanish grants: CONICET PIP 2461/01, UBACYT 20020100100945, PICT-2010-0525, Digiteo DIM 2009-36HD "Magix", ANR-2010-BLAN-0109-04 "LEDA", MTM2010-16051.

(C)2013 American Mathematical Society Reverts to public domain 28 years from publication 
$n$-dimensional complex projective space and by $\mathbb{P}_{\mathbb{R}}^{n}$ its real counterpart. We shall also use the following notations:

$$
\left\{F_{1}=0, \ldots, F_{p}=0\right\}:=S \text { and }\left\{F_{1}=0, \ldots, F_{p}=0\right\}_{\mathbb{R}}:=S_{\mathbb{R}} .
$$

We call the regular sequence $F_{1}, \ldots, F_{p}$ reduced if the ideal $\left(F_{1}, \ldots, F_{p}\right)$ generated in $\mathbb{Q}[X]$ is the ideal definition of the affine variety $S$, i.e., if $\left(F_{1}, \ldots, F_{p}\right)$ is radical. We call $\left(F_{1}, \ldots, F_{p}\right)$ strongly reduced if for any index $1 \leq k \leq p$ the ideal $\left(F_{1}, \ldots, F_{k}\right)$ is radical. Thus, a strongly reduced regular sequence is always reduced.

A point $x$ of $\mathbb{A}^{n}$ is called $\left(F_{1}, \ldots, F_{p}\right)$-regular if the Jacobian $J\left(F_{1}, \ldots, F_{p}\right):=$ $\left[\frac{\partial F_{j}}{\partial X_{k}}\right]_{\substack{1 \leq j \leq p \\ 1 \leq k \leq n}}$ has maximal rank $p$ at $x$. Observe, that for each reduced regular sequence $F_{1}, \ldots, F_{p}$ defining the variety $S$, the locus of $\left(F_{1}, \ldots, F_{p}\right)$-regular points of $S$ is the same. In this case we call an $\left(F_{1}, \ldots, F_{p}\right)$-regular point of $S$ simply regular (or smooth) or we say that $S$ is regular (or smooth) at $x$. The set $S_{\text {reg }}$ of regular points of $S$ is called the regular locus, whereas $S_{\text {sing }}:=S \backslash S_{\text {reg }}$ is called the singular locus of $S$. Remark that $S_{\text {reg }}$ is a non-empty open subvariety and $S_{\text {sing }}$ a proper closed subvariety of $S$. We say that a connected component $C$ of $S_{\mathbb{R}}$ is generically smooth if $C$ contains a smooth point.

We suppose now that there are natural numbers $d, L$ and $\ell$ and an essentially division-free arithmetic circuit $\beta$ in $\mathbb{Q}[X]$ with $p$ output nodes such that the following conditions are satisfied.

- The degrees $\operatorname{deg} F_{1}, \ldots, \operatorname{deg} F_{p}$ of the polynomials $F_{1}, \ldots, F_{p}$ are bounded by $d$.

- The $p$ output nodes of the arithmetic circuit $\beta$ represent the polynomials $F_{1}, \ldots, F_{p}$ by evaluation.

- The size and the non-scalar depth of the arithmetic circuit $\beta$ are bounded by $L$ and $\ell$, respectively.

For the terminology and basic facts concerning arithmetic circuits we refer to [11, 13, 22.

Suppose that the variables $X_{1}, \ldots, X_{n}$ are in generic position with respect to the variety $S$. Observe that we allow $S_{\mathbb{R}}$ to have singular points.

In this paper we design for each $1 \leq i \leq n-p$ a non-uniform deterministic or uniform probabilistic procedure $\Pi_{i}$ and an invariant $\delta_{i}$ satisfying the following specification.

(i) The invariant $\delta_{i}$ is a positive integer depending on $F_{1}, \ldots, F_{p}$ and having asymptotic order not exceeding $\left(n^{n} d\right)^{O(n)}$. We call $\delta_{i}$ the degree of the real interpretation of the equation system $F_{1}=0, \ldots, F_{p}=0$.

(ii) The algorithm $\Pi_{i}$ decides on input $\beta$ whether the variety $S$ contains a smooth real point and, if it is the case, produces for each generically smooth connected component of $S$ a suitably encoded real algebraic sample point.

(iii) In order to achieve this goal, the algorithm $\Pi_{i}$ performs on input $\beta$ a computation in $\mathbb{Q}$ with $L(n d)^{O(1)}\left(\min \left\{(n d)^{c n}, \delta_{i}\right\}\right)^{2}$ arithmetic operations (additions, subtractions, multiplications and divisions) which become organized in non-scalar depth $O\left(n(\ell+\log n d) \log \delta_{i}\right)$ with respect to the parameters of the arithmetic circuit $\beta$ (here $c>0$ is a suitable universal constant).

This is the outcome of our main result, namely Theorem 14 below and the three remarks following the theorem. 
Although we were not able to derive a better worst case bound as $\left(n^{n} d\right)^{O(n)}$ for the invariant $\delta_{i}$ (see Propositions 8 and 12 and Observation 11 below) the worst case complexity of the procedure $\Pi_{i}$ meets the already known extrinsic bound of $(n d)^{O(n)}$ for the elimination problem under consideration (compare the original papers [9, 12, 24, 30, 32, 35, 36] and the comprehensive book [10]).

The complexity of the procedure $\Pi_{i}$ depends polynomially on the extrinsic parameters $L, \ell, n$ and $d$ and on the degree $\delta_{i}$ of the real interpretation of the equation system $F_{1}=0, \ldots, F_{p}=0$ which represents an intrinsic parameter measuring the input size of our computational task. In this sense we say that the procedure $\Pi_{i}$ is of intrinsic complexity.

Since the complexity $L(n d)^{O(1)}\left(\min \left\{(n d)^{c n}, \delta_{i}\right\}\right)^{2}$ is polynomial in all its parameters, including the intrinsic parameter $\delta_{i}$, we say that the procedure $\Pi_{i}$ is pseudo-polynomial. In view of the main outcome of [27, 28], intrinsic complexity and pseudo-polynomiality constitute the best runtime behavior of $\Pi_{i}$ that can be expected for elimination algorithms implemented by rules of software engineering.

In the case that $S_{\mathbb{R}}$ is smooth and $F_{1}, \ldots, F_{p}$ is a strongly reduced regular sequence in $\mathbb{Q}[X]$ there already exist pseudo-polynomial algorithms of intrinsic complexity which solve the computational task of item (ii) above (see [1,3,4]). The same is true for the singular hypersurface case, namely $p:=1$, where $\left\{F_{1}=0\right\}_{\mathbb{R}}$ contains possibly singular points (see 6 6]). The methods developed in [1, 2, 4] cannot be applied directly when $S_{\mathbb{R}}$ is singular. To overcome this difficulty we consider in Section 3.1 two families of smooth incidence varieties which parametrize the so-called copolar varieties of $S$ introduced in Section 3.2 .

For a given full rank matrix $b \in \mathbb{A}^{(n-i) \times n}$, the corresponding copolar variety of $S$ is the Zariski closure of the set of all points $x$ of $S$ such that there exist $p$ rows of $b$ which generate the same linear space as the rows of the Jacobian of the equation system $F_{1}=0, \ldots, F_{p}=0$ at $x$.

The procedure $\Pi_{i}$ is based on a geometrical and computational analysis of the dual polar varieties of the two families of incidence varieties (see [3 5] for the notion of a dual polar variety). These geometric objects are called bipolar varieties of $S$. They become introduced in Section 4.1 Important for the worst case complexity of the procedure are the degree estimates for the bipolar varieties developed in Section 4.2 ,

\section{Preliminaries about POlar VARIETies}

Let notations be as in the Introduction. Let $F_{1}, \ldots, F_{p} \in \mathbb{Q}[X]$ be a reduced regular sequence defining a (non-empty) subvariety $S$ of $\mathbb{A}^{n}$ of pure codimension $p$.

Let $1 \leq i \leq n-p$ and let $a:=\left[a_{k, l}\right]_{\substack{1 \leq k \leq n-p-i+1 \\ 0 \leq l \leq n}}$ be a complex $((n-p-i+1) \times$ $(n+1))$-matrix and suppose that $a_{*}:=\left[a_{k, l}\right]_{\substack{1 \leq k \leq n-p-i+1 \\ 1 \leq l \leq n}}$ has maximal rank $n-$ $p-i+1$. In the case $\left(a_{1,0}, \ldots, a_{n-p-i+1,0}\right)=0$ we denote by $\underline{K}(a):=\underline{K}^{n-p-i}(a)$ and in the case $\left(a_{1,0}, \ldots, a_{n-p-i+1,0}\right) \neq 0$ by $\bar{K}(a):=\bar{K}^{n-p-i}(a)$ the $(n-p-i)-$ dimensional linear subvarieties of the projective space $\mathbb{P}^{n}$ which for $1 \leq k \leq n-$ $p-i+1$ are spanned by the points $\left(a_{k, 0}: a_{k, 1}: \cdots: a_{k, n}\right)$. In the first case we shall also use the notations $\underline{K}\left(a_{*}\right)$ and $\underline{K}^{n-p-i}\left(a_{*}\right)$ instead of $\underline{K}(a)$ and $\underline{K}^{n-p-i}(a)$.

The classic and the dual $i$ th polar varieties of $S$ associated with the linear varieties $\underline{K}(a)$ and $\bar{K}(a)$ are defined as the closures of the loci of the $\left(F_{1}, \ldots, F_{p}\right)$-regular 
points of $S$ where all $(n-i+1)$-minors of the respective polynomial $((n-i+1) \times n)-$ matrix

and

$$
\left[\begin{array}{ccc}
\frac{\partial F_{1}}{\partial X_{1}} & \cdots & \frac{\partial F_{1}}{\partial X_{n}} \\
\vdots & \vdots & \vdots \\
\frac{\partial F_{p}}{\partial X_{1}} & \cdots & \frac{\partial F_{p}}{\partial X_{n}} \\
a_{1,1} & \cdots & a_{1, n} \\
\vdots & \vdots & \vdots \\
a_{n-p-i+1,1} & \cdots & a_{n-p-i+1, n}
\end{array}\right]
$$
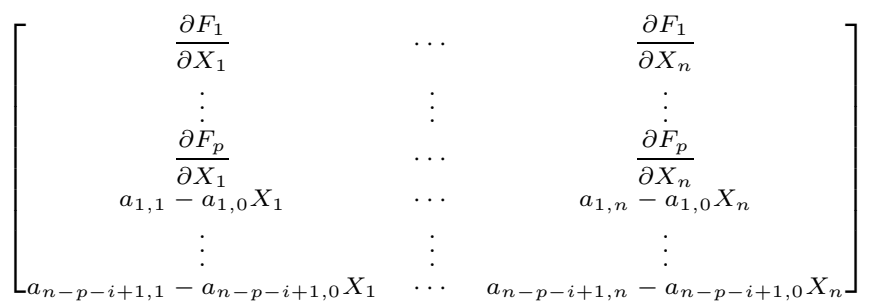

vanish. If $a$ is a real $((n-p-i+1) \times(n+1))$-matrix, we denote the real traces of the polar varieties $W_{\underline{K}(a)}(S)$ and $W_{\bar{K}(a)}(S)$ by

$$
W_{\underline{K}(a)}\left(S_{\mathbb{R}}\right):=W_{\underline{K}^{n-p-i}(a)}\left(S_{\mathbb{R}}\right):=W_{\underline{K}(a)}(S) \cap \mathbb{A}_{\mathbb{R}}^{n}
$$

and

$$
W_{\bar{K}(a)}\left(S_{\mathbb{R}}\right):=W_{\bar{K}^{n-p-i}(a)}\left(S_{\mathbb{R}}\right):=W_{\bar{K}(a)}(S) \cap \mathbb{A}_{\mathbb{R}}^{n}
$$

and call them the real polar varieties.

Observe that this definition of classic and dual polar varieties may be extended to the case that there is given a Zariski open subset $O$ of $\mathbb{A}^{n}$ such that the equations $F_{1}=0, \ldots, F_{p}=0$ intersect transversally at any of their common solutions in $O$ and that $S$ is now the locally closed subvariety of $\mathbb{A}^{n}$ given by

$$
S:=\left\{F_{1}=0, \ldots, F_{p}=0\right\} \cap O,
$$

which is supposed to be non-empty.

In Section 4 we shall need this extended definition of polar varieties in order to establish the notion of a bipolar variety of a given complete intersection. For the moment let us suppose again that $S$ is the closed subvariety of $\mathbb{A}^{n}$ defined by the reduced regular sequence $F_{1}, \ldots, F_{p}$.

In [3] and [4] we have introduced the notion of dual polar varieties of $S$ (and $S_{\mathbb{R}}$ ) and motivated by geometric arguments the calculatory definition of these objects. Moreover, we have shown that, for a complex $((n-p-i+1) \times(n+1))$-matrix $a=\left[a_{k, l}\right]_{\substack{1 \leq k \leq n-p-i+1 \\ 0 \leq l \leq n}}$ with $\left[a_{k, l}\right]_{\substack{1 \leq k \leq n-p-i+1 \\ 1 \leq l \leq n}}$ generic, the polar varieties $W_{\underline{K}(a)}(S)$ and $W_{\bar{K}(a)}(S)$ are either empty or of pure codimension $i$ in $S$. Further, we have shown that $W_{\underline{K}(a)}(S)$ and $W_{\bar{K}(a)}(S)$ are normal and Cohen-Macaulay (but not necessarily smooth) at any of their $\left(F_{1}, \ldots, F_{p}\right)$-regular points (see [5, Corollary 2] and Section 3.1). This motivates the consideration of the so-called generic polar varieties $W_{\underline{K}(a)}(S)$ and $W_{\bar{K}(a)}(S)$, associated with complex $((n-p-i+1) \times(n+1))-$ matrices $a$ which are generic in the above sense, as invariants of the complex variety $S$ (independently of the given equation system $\left.F_{1}=0, \ldots, F_{p}=0\right)$. However, when a generic $((n-p-i+1) \times(n+1))$-matrix $a$ is real, we cannot consider $W_{\underline{K}(a)}\left(S_{\mathbb{R}}\right)$ and $W_{\bar{K}(a)}\left(S_{\mathbb{R}}\right)$ as invariants of the real variety $S_{\mathbb{R}}$, since for suitable real generic 
$((n-p-i+1) \times(n+1))$-matrices these polar varieties may turn out to be empty, whereas for other real generic matrices they may contain points (see [5] Theorem 1 and Corollary 2] and [8, Theorem 8 and Corollary 9]).

For our use of the word "generic" we refer to [5] Definition 1].

In case that $S_{\mathbb{R}}$ is smooth and $a$ is a real $((n-p-i+1) \times(n+1))$-matrix, the real dual polar variety $W_{\bar{K}(a)}\left(S_{\mathbb{R}}\right)$ contains at least one point of each connected component of $S_{\mathbb{R}}$, whereas the classic (complex or real) polar varieties $W_{\underline{K}(a)}(S)$ and $W_{\underline{K}(a)}\left(S_{\mathbb{R}}\right)$ may be empty (see [3] and [4, Proposition 2]).

Polar varieties have a long history in algebraic geometry which goes back to Severi [39] and Todd [43,44] in the 1930s. Originally they were used to establish numerical formulas in order to classify singular algebraic varieties by their intrinsic geometric character or to formulate a manageable local equisingularity criterion which implies the Whitney conditions for analytic varieties. About 10 years ago they also became a fundamental tool for the design of pseudo-polynomial computer procedures with intrinsic complexity bounds which find for a given complete intersection variety $S$ with a smooth real trace $S_{\mathbb{R}}$ algebraic sample points for each connected component of $S_{\mathbb{R}}$. For details we refer to [42] and [5].

\section{Copolar incidence varieties}

3.1. Two families of copolar incidence varieties. Let $d, n, p$ and $i$ be natural numbers with $1 \leq p \leq n$ and $1 \leq i \leq n-p$ and let $X:=\left(X_{1}, \ldots, X_{n}\right)$, $B:=\left[B_{k, l}\right]_{\substack{1 \leq k \leq n-i \\ 1 \leq l \leq n}}, \Lambda:=\left[\Lambda_{r, s}\right]_{1 \leq r, s \leq p}$ and $\Theta:=\left[\Theta_{k, r}\right]_{\substack{1 \leq k \leq n-i \\ 1 \leq r \leq p}}$ be matrices of indeterminates over $\mathbb{C}$.

For the rest of this paper we fix a strongly reduced regular sequence $F_{1}, \ldots, F_{p} \in$ $\mathbb{Q}[X]$. Let $d:=\max \left\{\operatorname{deg} F_{s} \mid 1 \leq s \leq p\right\}$, where $\operatorname{deg} F_{s}$ denotes the degree of the polynomial $F_{s}$. We denote by $F:=\left(F_{1}, \ldots, F_{p}\right)$ the sequence of these polynomials and by $J(F):=\left[\frac{\partial F_{s}}{\partial X_{l}}\right]_{\substack{1 \leq s \leq p \\ 1 \leq l \leq n}}$ the Jacobian of $F$. Observe that the rank of $J(F)$ is generically $p$ on any irreducible component of the complex variety $S:=\left\{F_{1}=\right.$ $\left.\cdots=F_{p}=0\right\}$. We write $J(F)^{T}$ for the transposed matrix of $J(F)$ and for any point $x \in \mathbb{A}^{n}$ we denote by rk $J(F)(x)$ the rank of the complex matrix $J(F)(x)$.

We are now going to introduce two families of varieties which we shall call copolar incidence varieties. In order to define the first one we consider in the ambient space,

$$
\mathbb{T}_{i}:=\mathbb{A}^{n} \times \mathbb{A}^{(n-i) \times n} \times \mathbb{A}^{p \times p} \times \mathbb{A}^{(n-i) \times p},
$$

the $\mathbb{Q}$-definable locally closed incidence variety

$$
H_{i}:=\left\{(x, b, \lambda, \vartheta) \in \mathbb{T}_{i} \mid x \in S, \text { rk } b=n-i, \text { rk } \vartheta=p, J(F)(x)^{T} \lambda+b^{T} \vartheta=0\right\} .
$$

Observe that the isomorphy class of $H_{i}$ does not depend on the choice of the generators $F_{1}, \ldots, F_{p}$ of the vanishing ideal of $S$. The canonical projection of $\mathbb{T}_{i}$ onto $\mathbb{A}^{n}$ maps $H_{i}$ into $S$.

We are now going to state and prove three facts, namely Lemma 11 and Propositions 2 and 3 below, which will be fundamental in the sequel.

Lemma 1. Let $(x, b, \lambda, \vartheta)$ be a point of $H_{i}$. Then $x$ belongs to $S_{\mathrm{reg}}$ and $\lambda$ is a regular complex $(p \times p)$-matrix. Moreover, the canonical projection of $\mathbb{T}_{i}$ onto $\mathbb{A}^{n}$ maps $H_{i}$ onto $S_{\mathrm{reg}}$ and $\left(H_{i}\right)_{\mathbb{R}}$ onto $\left(S_{\mathbb{R}}\right)_{\mathrm{reg}}$.

Proof. Let $(x, b, \lambda, \vartheta)$ be a point of $H_{i}$. Then $b$ and $\vartheta$ are complex full rank matrices of size $(n-i) \times n$ and $(n-i) \times p$, respectively. Therefore $b^{T} \vartheta$ is a complex full 
rank matrix of size $n \times p$. From $J(F)(x)^{T} \lambda+b^{T} \vartheta=0$ we deduce that the complex $(n \times p)$-matrix $J(F)(x)^{T} \lambda$ and the matrix $\lambda$ have rank $p$. This implies that the rank of $J(F)(x)$ is $p$. Since $x$ belongs to $S$ we conclude that $S$ is smooth at $x$. Thus we have $x \in S_{\text {reg }}$ and $\lambda$ is a regular complex $(p \times p)$-matrix. By the way, we have shown that the canonical projection of $\mathbb{T}_{i}$ onto $\mathbb{A}^{n}$ maps $H_{i}$ into $S_{\text {reg }}$.

Consider now an arbitrary point $x \in S_{\text {reg }}$. Without loss of generality we may assume that the first $p$ columns of $J(F)(x)$ are $\mathbb{C}$-linearly independent. Let $\lambda$ be the $(p \times p)$-identity matrix $I_{p}$. Furthermore, let

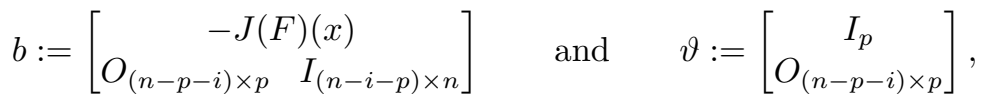

where $O_{(n-p-i) \times p}$ denotes the $((n-p-i) \times p)$-zero matrix.

Then $b$ and $\vartheta$ are full rank matrices which satisfy the condition $J(F)(x)^{T} \lambda+$ $b^{T} \vartheta=0$. Since $x$ belongs to $S$, we conclude that $(x, b, \lambda, \vartheta)$ is an element of $H_{i}$ which becomes mapped onto $x$ under the canonical projection of $\mathbb{T}_{i}$ onto $\mathbb{A}^{n}$. In particular, if $x \in\left(S_{\mathbb{R}}\right)_{\mathrm{reg}}$, then $\lambda, b$ and $\vartheta$ are real matrices and $(x, b, \lambda, \vartheta)$ belongs to $\left(H_{i}\right)_{\mathbb{R}}$. This implies that the canonical projection of $\mathbb{T}_{i}$ onto $\mathbb{A}^{n}$ maps $H_{i}$ onto $S_{\text {reg }}$ and $\left(H_{i}\right)_{\mathbb{R}}$ onto $\left(S_{\mathbb{R}}\right)_{\text {reg }}$.

Proposition 2. Let $D_{i}$ be the closed subvariety of $\mathbb{T}_{i}$ defined by the conditions $r k B<n-i$ or $r k \Theta<p$. Then the polynomial equations

$$
\begin{gathered}
F_{1}(X)=\cdots=F_{p}(X)=0, \\
\sum_{1 \leq s \leq p} \Lambda_{r, s} \frac{\partial F_{s}}{\partial X_{l}}(X)+\sum_{\substack{1 \leq k \leq n-i \\
1 \leq n, l}} B_{k, r}=0, \\
1 \leq r \leq p, 1 \leq l \leq n,
\end{gathered}
$$

intersect transversally at any of their common solutions in $\mathbb{T}_{i} \backslash D_{i}$. Moreover, $H_{i}$ is exactly the set of solutions of the polynomial equation system (11) outside of the locus $D_{i}$.

In particular, $H_{i}$ is an equidimensional algebraic variety which is smooth and of dimension $n(n-i+1)+p(p-i-1) \geq 0$.

Proof. One sees immediately that a point $(x, b, \lambda, \vartheta) \in \mathbb{T}_{i}$ belongs to $H_{i}$ if and only if it is a solution of the polynomial equation system (10) outside of the locus $D_{i}$. The Jacobian of system (11) at such a point $(x, b, \lambda, \vartheta)$ has the following form:

$$
\mathcal{L}_{x}:=\left[\begin{array}{cccccccc}
J(F)(x) & O_{p \times p} & \cdots & O_{p \times p} & O_{p \times(n-i)} & \cdots & O_{p \times(n-i)} & O_{p \times n(n-i)} \\
& J(F)(x)^{T} & \cdots & O_{n \times p} & b^{T} & \cdots & O_{n \times(n-i)} & D^{(1)} \\
* & \vdots & \ddots & \vdots & \vdots & \ddots & \vdots & \vdots \\
& O_{n \times p} & \cdots & J(F)(x)^{T} & O_{n \times(n-i)} & \cdots & b^{T} & D^{(p)}
\end{array}\right],
$$

where $D^{(r)}, 1 \leq r \leq p$, is the complex $(n \times n(n-i))$-matrix

$$
D^{(r)}:=\left[\begin{array}{cccccc}
\vartheta_{1, r} & \cdots & \vartheta_{n-i, r} & \cdots & O_{1 \times(n-i)} & \\
& \vdots & \ddots & & \vdots & \\
O_{1 \times(n-i)} & \cdots & \vartheta_{1, r} & \cdots & \vartheta_{n-i, r}
\end{array}\right] .
$$

From Lemma 1 we conclude that the $(p \times n)$-matrix $J(F)(x)$ has maximal rank $p$. Since the matrix $\vartheta$ has full rank we infer that the $(n p \times n(n-i))$-submatrix of 
$\mathcal{L}_{x}$ built up by $D^{(1)}, \ldots, D^{(r)}$ has rank $n p$. This implies that the Jacobian $\mathcal{L}_{x}$ has full rank. Therefore, the $(n+1) p$ equations of system (11) intersect transversally at $(x, b, \lambda, \vartheta)$ and the algebraic variety $H_{i}$ is smooth and of dimension

$$
n+(n-i) n+p^{2}+(n-i) p-(n+1) p=n(n-i+1)+p(p-i-1)
$$

at this point. Thus $H_{i}$ is an equidimensional variety which is empty or smooth and of dimension $n(n-i+1)+p(p-i-1)$ (observe that $1 \leq i \leq n-p$ implies $n(n-i+1)+p(p-i-1) \geq 0)$. From Lemma 1 we deduce that $H_{i}$ is not empty.

For algorithmic applications Proposition 2 contains too many open conditions, namely the conditions $\operatorname{rk} B=n-i$ and $\operatorname{rk} \Theta=p$. By means of a suitable specialization of the matrices $B$ and $\Theta$ we are going to eliminate these open conditions. However, we have to take care that this specialization process does not exclude many smooth points of the variety $S$. The following result, namely Proposition 3 below seems to represent a fair compromise. We shall need it later for the task of finding smooth points of $S$. For the formulation of this proposition we need some notation.

Let $\mathbf{B}$ and $\boldsymbol{\Theta}$ be the following matrices:

$$
\mathbf{B}:=\left[\begin{array}{ccc}
B_{1, n-i+1} & \cdots & B_{1, n} \\
\vdots & \cdots & \vdots \\
B_{p, n-i+1} & \cdots & B_{p, n}
\end{array}\right] \quad \text { and } \quad \boldsymbol{\Theta}:=\left[\begin{array}{ccc}
\Theta_{p+1,1} & \cdots & \Theta_{p+1, p} \\
\vdots & \cdots & \vdots \\
\Theta_{n-i, 1} & \cdots & \Theta_{n-i, p}
\end{array}\right] \text {. }
$$

Let $\sigma$ be a permutation of the set $\{1, \ldots, n\}$ (in symbols, $\sigma \in \operatorname{Sym}(n)$ ) and apply $\sigma$ to the columns of the $((n-i) \times n)$-matrix

$$
\left[\begin{array}{ccc}
I_{p} & O_{p \times(n-p-i)} & \mathbf{B} \\
O_{(n-p-i) \times p} & I_{n-p-i} & O_{(n-p-i) \times i}
\end{array}\right] .
$$

In this way we obtain a $((n-i) \times n)$-matrix which we denote by $\mathbf{B}_{i, \sigma}$. Furthermore, let

$$
\boldsymbol{\Theta}_{i}:=\left[\begin{array}{l}
I_{p} \\
\boldsymbol{\Theta}
\end{array}\right] \quad \text { and } \quad \Delta_{\sigma}:=\operatorname{det}\left[\frac{\partial F_{s}}{\partial X_{\sigma(r)}}\right]_{1 \leq s, r \leq p} .
$$

If we specialize in $\mathbf{B}_{i, \sigma}$ the submatrix $\mathbf{B}$ to $b \in \mathbb{A}^{p \times i}$ and in $\boldsymbol{\Theta}_{i}$ the submatix $\boldsymbol{\Theta}$ to $\vartheta \in \mathbb{A}^{(n-p-i) \times p}$, then the resulting complex matrices become denoted by $b_{i, \sigma}$ and $\vartheta_{i}$, respectively.

We consider now in the ambient space

$$
\mathbb{F}_{i}:=\mathbb{A}^{n} \times \mathbb{A}^{p \times i} \times \mathbb{A}^{p \times p} \times \mathbb{A}^{(n-p-i) \times p}
$$

a copolar incidence variety of a more restricted type, namely

$$
H_{i, \sigma}:=\left\{(x, b, \lambda, \vartheta) \in \mathbb{F}_{i} \mid x \in S, J(F)(x)^{T} \lambda+b_{i, \sigma}^{T} \vartheta_{i}=0\right\} .
$$

Observe that $H_{i, \sigma}$ is a $\mathbb{Q}$-definable closed subvariety of $\mathbb{F}_{i}$ whose isomorphy class does not depend on the choice of the polynomials $F_{1}, \ldots, F_{p}$ of the vanishing ideal of $S$.

In the statement of the next result we make use of the Kronecker symbol $\delta_{r, l}, 1 \leq$ $r, l \leq p$ which is defined by $\delta_{r, l}:=0$ for $r \neq l$ and $\delta_{r, r}:=1$.

Proposition 3. Let notations and definitions be as before. For the sake of simplicity assume that $\sigma$ is the identity permutation of Sym $(n)$. Then the polynomial 
equations

$$
\begin{gathered}
F_{1}=0, \ldots, F_{s}=0, \\
\sum_{1 \leq s \leq p} \Lambda_{r, s} \frac{\partial F_{s}}{\partial X_{l}}(X)+\delta_{r, l}=0, \quad 1 \leq r \leq p, 1 \leq l \leq p, \\
\sum_{1 \leq s \leq p} \Lambda_{r, s} \frac{\partial F_{s}}{\partial X_{l}}(X)+\Theta_{l, r}=0,1 \leq r \leq p, p<l \leq n-i, \\
\sum_{1 \leq s \leq p} \Lambda_{r, s} \frac{\partial F_{s}}{\partial X_{l}}(X)+B_{r, l}=0, \quad 1 \leq r \leq p, n-i<l \leq n,
\end{gathered}
$$

intersect transversally at any of their common solutions in $\mathbb{F}_{i}$. Moreover, $H_{i, \sigma}$ is exactly the set of solutions of system (2). In particular, $H_{i, \sigma}$ is a closed equidimensional algebraic variety which is empty or smooth and of dimension $n-p$.

The image of $H_{i, \sigma}$ under the canonical projection of $\mathbb{F}_{i}$ onto $\mathbb{A}^{n}$ is the set of (smooth) points of $S$ where $\Delta_{\sigma}$ does not vanish. For each real point $x \in S$ with $\Delta_{\sigma}(x) \neq 0$ there exists a real point $(x, b, \lambda, \vartheta)$ of $H_{i, \sigma}$.

Proof. From the matrix identities

$$
\mathbf{B}_{\mathbf{i}, \sigma}{ }^{T} \boldsymbol{\Theta}_{\mathbf{i}}=\left[\begin{array}{cc}
I_{p} & O_{p \times(n-p-i)} \\
O_{(n-p-i) \times p} & I_{n-p-i} \\
\mathbf{B}^{T} & O_{i \times(n-p-i)}
\end{array}\right]\left[\begin{array}{l}
I_{p} \\
\boldsymbol{\Theta}
\end{array}\right]=\left[\begin{array}{c}
I_{p} \\
\boldsymbol{\Theta} \\
\mathbf{B}^{T}
\end{array}\right]=\left[\begin{array}{ccc}
1 & \cdots & 0 \\
\vdots & \cdots & \vdots \\
0 & \cdots & 1 \\
\Theta_{p+1,1} & \cdots & \Theta_{p+1, p} \\
\vdots & \cdots & \vdots \\
\Theta_{n-i, 1} & \cdots & \Theta_{n-i, p} \\
B_{1, n-i+1} & \cdots & B_{p, n-i+1} \\
\vdots & \cdots & \vdots \\
B_{1, n} & \cdots & B_{p, n}
\end{array}\right]
$$

one deduces easily that a point $(x, b, \lambda, \vartheta)$ of $\mathbb{F}_{i}$ belongs to $H_{i, \sigma}$ if and only if it is a solution of the polynomial equation system (2).

Let $(x, b, \lambda, \vartheta)$ be such a point of $H_{i, \sigma}$. Then system (2) implies $\Delta_{\sigma}(x) \neq 0$ and its Jacobian may be organized as the matrix

$$
\mathcal{L}_{x}^{(i, \sigma)}:=\left[\begin{array}{ccccccc}
J(F)(x) & O_{p \times p} & \cdots & O_{p \times p} & O_{p \times(n-p)} & \cdots & O_{p \times(n-p)} \\
& J(F)(x)^{T} & \cdots & O_{n \times p} & Z & \cdots & O_{n \times(n-p)} \\
* & \vdots & \ddots & \vdots & \vdots & \ddots & \vdots \\
& O_{n \times p} & \cdots & J(F)(x)^{T} & O_{n \times(n-p)} & \cdots & Z
\end{array}\right]
$$

with

$$
Z:=\left[\begin{array}{cc}
O_{p \times(n-p-i)} & O_{p \times i} \\
I_{n-p-i} & O_{(n-p-i) \times i} \\
O_{i \times(n-p-i)} & I_{i}
\end{array}\right] .
$$

From $\Delta_{\sigma}(x) \neq 0$ we deduce that $J(F)(x)$ has rank $p$. Thus $\mathcal{L}_{x}^{(i, \sigma)}$ has full rank. Therefore, the $(n+1) p$ equations of system (2) intersect transversally at 
$(x, b, \lambda, \vartheta)$ and the algebraic variety $H_{i, \sigma}$ is smooth and of dimension $n+p i+p^{2}+$ $(n-p-i) p-(n+1) p=n-p$ at this point. Thus $H_{i, \sigma}$ is an equidimensional variety which is empty or smooth of dimension $n-p$. For any point $(x, b, \lambda, \vartheta)$ of $H_{i, \sigma}$ we have $\Delta_{\sigma}(x) \neq 0$ and, in particular, $S$ is smooth at $x$.

On the other hand, for $x \in S$ with $\Delta_{\sigma}(x) \neq 0$ we may consider

$$
\lambda:=-\left[\frac{\partial F_{s}(x)}{\partial X_{l}}\right]_{1 \leq s, l \leq p}^{-1}, \vartheta:=-\left[\frac{\partial F_{s}(x)}{\partial X_{l}}\right]_{\substack{1 \leq s \leq p \\ p<l \leq n-i}}^{T} \cdot \lambda \text { and } b:=-\left[\frac{\partial F_{s}(x)}{\partial X_{l}}\right]_{\substack{1 \leq s \leq p \\ n-i \leq l \leq n}}^{T} \cdot \lambda .
$$

Then the corresponding point $(x, b, \lambda, \vartheta)$ belongs to $H_{i, \sigma}$. Moreover, for $x$ real we have that $b, \lambda, \vartheta$ are also real and therefore $(x, b, \lambda, \vartheta)$ is a real point of $H_{i, \sigma}$.

In the sequel we shall refer to $H_{i}$ and $H_{i, \sigma}$ as the copolar incidence varieties of $S:=\left\{F_{1}=\cdots=F_{p}=0\right\}$ associated with the indices $1 \leq i \leq n-p$ and $\sigma \in \operatorname{Sym}(n)$.

The notion of a copolar incidence variety is inspired by the Room-Kempf desingularization of determinantal varieties $[33,37$.

3.2. Copolar varieties. Let notations and assumptions be as in previous section and let $b \in \mathbb{A}^{(n-i) \times n}$ be a full rank matrix. We observe that the set

$$
\widetilde{V}_{b}(S):=\left\{x \in S \mid \exists(\lambda, \vartheta) \in \mathbb{A}^{p \times p} \times \mathbb{A}^{(n-p) \times p}: \operatorname{rk} \vartheta=p \text { and }(x, b, \lambda, \vartheta) \in H_{i}\right\}
$$

does not depend on the choice of the generators $F_{1}, \ldots, F_{p}$ of the vanishing ideal of $S$. We call the Zariski closure in $\mathbb{A}^{n}$ of $\widetilde{V}_{b}(S)$ the copolar variety of $S$ associated with the matrix $b$ and we denote it by $V_{b}(S)$. From the argumentation at the end of the proof of Lemma 1 we deduce $\widetilde{V}_{b}(S)=V_{b}(S) \cap S_{\text {reg }}$.

Observe that a point $x$ of $S$ belongs to $\widetilde{V}_{b}(S)$ if and only if there exist $p$ rows of the $((n-i) \times n)$-matrix $b$ which generate the same affine linear space as the rows of the Jacobian $J(F)$ at $x$. In case $p:=1$ and $F:=F_{1}$ the copolar variety $V_{b}(\{F=0\})$ coincides with the $i$ th classic polar variety $W_{\underline{K}^{n-1-i}(\underline{b})}(\{F=0\})$ of the complex hypersurface $\{F=0\}$ (here $\underline{b}$ denotes the $((n-i) \times(n+1))$-matrix whose column number zero is a null-vector, whereas the columns numbered $1, \ldots, n$ are the corresponding columns of $b$ ).

Proposition 4. If $b \in \mathbb{A}^{(n-i) \times n}$ is a generic matrix, then the copolar variety $V_{b}(S)$ is empty or an equidimensional closed subvariety which is smooth at any point of $V_{b}(S) \cap S_{\text {reg }}$ and has (non-negative) dimension $n-(i+1) p$.

Proof $($ Sketch $)$. We consider in the ambient space $\widetilde{\mathbb{F}}_{i}:=\mathbb{A}^{n} \times \mathbb{A}^{(n-i) \times n} \times \mathbb{A}^{(n-i) \times p}$ the $\mathbb{Q}$-definable locally closed incidence variety

$$
\widetilde{H}_{i}:=\left\{(x, b, \vartheta) \in \widetilde{\mathbb{F}}_{i} \mid x \in S, \text { rk } b=n-i, \text { rk } \vartheta=p, J(F)(x)^{T}+b^{T} \vartheta=0\right\} .
$$

Using the same argument as in Proposition 2 we see that $\widetilde{H}_{i}$ is non-empty, equidimensional of dimension $n(n-i+1)-(i+1) p \geq 0$ and smooth. Let $\pi: \widetilde{H}_{i} \mapsto \mathbb{A}^{(n-i) \times n}$ be the morphism induced by the canonical projection of $\widetilde{\mathbb{F}}_{i}$ onto $\mathbb{A}^{(n-i) \times n}$. Notice that for any full rank matrix $b \in \mathbb{A}^{(n-i) \times n}$ the $\pi$-fiber of $b$ is isomorphic to $V_{b}(S) \cap S_{\text {reg }}$ as algebraic variety. Suppose now that $\pi$ is dominating. From Sard's Theorem (see [16, 40]) we deduce that for a generic $b \in \mathbb{A}^{(n-i) \times n}$ the $\pi$-fiber of $b$ and hence $V_{b}(S) \cap S_{\text {reg }}$, are non-empty, equidimensional of dimension $n-(i+1) p \geq 0$ and smooth. If $\pi$ is not dominating, then we see by the same argument that $V_{b}(S)$ is empty. 
Observe that for a generic $b \in \mathbb{A}^{(n-i) \times n}$ the emptiness or non-emptiness and in the latter case also the geometric degree of the copolar variety $V_{b}(S)$ is an invariant of the variety $S$.

The incidence varieties $H_{i}$ and $H_{i, \sigma}$ may be interpreted as suitable algebraic families of copolar varieties. In [8] we considered in the case $p:=1$ three analogous incidence varieties which turned out to be algebraic families of dual polar varieties. Here we have a similar situation since in the hypersurface case, namely in the case $p:=1$, the copolar varieties are classic polar varieties.

\section{BIPOLAR VARIETIES}

4.1. Definition and basic properties of bipolar varieties. In order to measure the complexity of the real point finding procedures of this paper for complete intersection varieties, we consider for $1 \leq p \leq n, 1 \leq i \leq n-p$ and $\sigma \in \operatorname{Sym}(n)$ the generic dual polar varieties of the copolar incidence varieties $H_{i}$ and $H_{i, \sigma}$. In analogy to the hypersurface case tackled in [8], we call them the large and the small bipolar varieties of $S$.

Definition 5. The bipolar varieties $\mathfrak{B}_{(i, j)}$ and $\mathcal{B}_{(i, \sigma, j)}$ are defined as follows:

- for $1 \leq j \leq n(n-i+1)+p(p-i-1)$ let $\mathfrak{B}_{(i, j)}$ be a $(n(n-i+1)+$ $p(p-i-1)-j+1)$ th generic dual polar variety of $H_{i}$ and,

- for $1 \leq j \leq n-p$ and $\sigma \in \operatorname{Sym}(n)$ let $\mathcal{B}_{(i, \sigma, j)}$ be a $(n-p-j+1)$ th generic dual polar variety of $H_{i, \sigma}$.

We call $\mathfrak{B}_{(i, j)}$ the large and $\mathcal{B}_{(i, \sigma, j)}$ the small bipolar variety of $S$, respectively.

The bipolar varieties $\mathfrak{B}_{(i, j)}$ and $\mathcal{B}_{(i, \sigma, j)}$ are well-defined geometric objects which depend on the system $F_{1}(X)=\cdots=F_{p}(X)=0$, although the copolar incidence varieties $H_{i}$ is not closed (compare the definition of the notion of polar variety in Section 2, where we have taken care of this situation). Moreover, our notation is justified because we are only interested in invariants like the dimension and the degree of our bipolar varieties and these are independent of the particular (generic) choice of the linear projective varieties used to define the bipolar varieties.

From Propositions 2] and 3] and [5, Corollary 2], we deduce that $\mathfrak{B}_{(i, j)}$ and $\mathcal{B}_{(i, \sigma, j)}$ are empty or equidimensional of dimension $j-1$ and Cohen-Macaulay and normal at any point of $\mathfrak{B}_{(i, j)} \cap H_{i}$ and $\mathcal{B}_{(i, \sigma, j)} \cap H_{i, \sigma}$.

Now let $1 \leq j \leq n(n-i+1)+p(p-i-1), a_{0} \in \mathbb{A}^{j}$ with $a_{0} \neq 0, a_{*} \in$ $\mathbb{A}^{j \times(n(n-i+1)+p(n+p-1))}$ generic and $a:=\left[a_{0}^{T}, a_{*}\right]$. Furthermore, let $T_{a}^{(i, j)}$ be the polynomial $(((n+1) p+j) \times(n(n-i+1)+p(n+p-i)))$-matrix whose first $(n+1) p$ rows constitute the Jacobian of the system (11) of Section 3 and whose remaining $j$ rows are built as in Section 2 in order to define the $(n(n-i+1)+p(p-i-1)-j+1)$ th dual polar variety of $H_{i}$ associated with the linear variety $\bar{K}(a)$. Then $\mathfrak{B}_{(i, j)}$ is the Zariski closure in $\mathbb{T}_{i}$ of the set of all points $(x, b, \lambda, \vartheta) \in H_{i}$ where $T_{a}^{(i, j)}(x, b, \lambda, \vartheta)$ does not have full rank.

By $\widetilde{T}_{a}^{(i, j)}$ we denote the polynomial $(((n+1) p+j-1) \times(n(n-i+1)+p(n+p-i)))-$ matrix consisting of all rows of $T_{a}^{(i, j)}$ except the last one.

Observe that the large bipolar varieties of $S$ form a chain of equidimensional varieties

$$
\overline{H_{i}} \supsetneqq \mathfrak{B}_{(i, n(n-i+1)+p(p-i-1))} \supset \cdots \supset \mathfrak{B}_{(i, 1)} .
$$


The variety $\mathfrak{B}_{(i, 1)}$ is empty or zero-dimensional. If $\mathfrak{B}_{(i, 1)}$ is non-empty, then the chain is strictly decreasing. We define $\mathfrak{B}_{(i, 0)}:=\emptyset$.

For $t \in \mathbb{N}^{j-1}$ with $t:=\left(t_{1}, \ldots, t_{j-1}\right)$ and

$$
(n+1) p<t_{1}<\cdots<t_{j-1} \leq n(n-i+1)+p(n+p-i)
$$

we denote by $m_{(i, j ; t)}$ the $((n+1) p+j-1)$-minor of $\widetilde{T}_{a}^{(i, j)}$ which corresponds to the first $(n+1) p$ columns and the columns numbered $t_{1}, \ldots, t_{j-1}$ of $\widetilde{T}_{a}^{(i, j)}$. Moreover, for

$$
(n+1) p<k_{1}<\cdots<k_{n(n-i+1)+p(p-i-1)-j+1} \leq n(n-i+1)+p(n+p-i)
$$

different from $t_{1}, \ldots, t_{j-1}$ and $1 \leq h \leq n(n-i+1)+p(p-i-1)-j+1$ we denote by $M_{h}^{(i, j ; t)}$ the $((n+1) p+j)$-minor of $T_{a}^{(i, j)}$ which corresponds to the first $(n+1) p$ columns of $T_{a}^{(i, j)}$ and the columns numbered $t_{1}, \ldots, t_{j-1}$ and $k_{h}$. Observe $\operatorname{deg} M_{h}^{(i, j ; t)} \leq(n+1) p d+j$.

Finally, for $t^{\prime} \in \mathbb{N}^{n-i}$ and $t^{\prime \prime} \in \mathbb{N}^{p}$ with $t^{\prime}:=\left(t_{1}^{\prime}, \ldots, t_{n-i}^{\prime}\right), t^{\prime \prime}:=\left(t_{1}^{\prime \prime}, \ldots, t_{p}^{\prime \prime}\right)$ and

$$
1 \leq t_{1}^{\prime}<\cdots<t_{n-i}^{\prime} \leq n \text { and } 1 \leq t_{1}^{\prime \prime}<\cdots<t_{p}^{\prime \prime} \leq n-i,
$$

let $B_{\left(i, t^{\prime}\right)}$ and $\Theta_{\left(i, t^{\prime \prime}\right)}$ be the $(n-i)$ - and $p$-minors of the matrices $B$ and $\Theta$ which correspond to the columns $t_{1}^{\prime}, \ldots, t_{n-i}^{\prime}$ and rows $t_{1}^{\prime \prime}, \ldots, t_{p}^{\prime \prime}$ of $B$ and $\Theta$, respectively. By induction on $n(n-i+1)+p(p-i-1, \ldots, 1)$ one sees easily that for any point $(x, b, \lambda, \vartheta)$ of $\mathfrak{B}_{(i, j)} \cap H_{i} \backslash \mathfrak{B}_{(i, j-1)}$ there exist suitable vectors $t \in \mathbb{N}^{j-1}, t^{\prime} \in \mathbb{N}^{n-i}$ and $t^{\prime \prime} \in \mathbb{N}^{p}$ with $m_{(i, j ; t)} B_{\left(i, t^{\prime}\right)} \Theta_{\left(i, t^{\prime \prime}\right)}(x, b, \lambda, \vartheta) \neq 0$.

Now Proposition 2 and Propositions 6 and 8 of [3,4] imply that the equations of system (11) and the equations

$$
M_{1}^{(i, j ; t)}=0, \ldots, M_{n(n-i+1)+p(p-i-1)-j+1)}^{(i, j ; t)}=0
$$

intersect transversally at $(x, b, \lambda, \vartheta)$. In particular, the corresponding polynomials form a regular sequence in

$$
\mathbb{Q}[X, B, \Lambda, \Theta]_{m_{(i, j ; t)} B_{\left(i, t^{\prime}\right)}} \Theta_{\left(i, t^{\prime \prime}\right)}
$$

and they define the large bipolar variety $\mathfrak{B}_{(i, j)}$ outside of the locus given by

$$
m_{(i, j ; t)} B_{\left(i, t^{\prime}\right)} \Theta_{\left(i, t^{\prime \prime}\right)}=0 .
$$

Finally, observe that there exist $\left(\begin{array}{c}n(n-i+1)+p(p-i-1) \\ j-1\end{array}\right),\left(\begin{array}{c}n \\ n-i\end{array}\right)$ and $\left(\begin{array}{c}n-i \\ p\end{array}\right)$ possible choices of the vectors $t \in \mathbb{N}^{j-1}, t^{\prime} \in \mathbb{N}^{n-i}$ and $t^{\prime \prime} \in \mathbb{N}^{p}$, respectively. This yields $\left(\begin{array}{c}n(n-i+1)+p(p-i-1) \\ j-1\end{array}\right)\left(\begin{array}{c}n \\ n-i\end{array}\right)\left(\begin{array}{c}n-i \\ p\end{array}\right)$ possible choices of vectors $\left(t, t^{\prime}, t^{\prime \prime}\right) \in \mathbb{N}^{j-1} \times \mathbb{N}^{n-i} \times$ $\mathbb{N}^{p}$.

These considerations entail the following statement.

Proposition 6. Let notation be as above and let $t \in \mathbb{N}^{j-i}, t^{\prime} \in \mathbb{N}^{n-i}$ and $t^{\prime \prime} \in \mathbb{N}^{p}$ be suitable vectors. Further, let $D_{\left(i, j ; t, t^{\prime}, t^{\prime \prime}\right)}$ be the closed variety of $\mathbb{T}_{i}$ defined by the condition $m_{(i, j ; t)} B_{\left(i, t^{\prime}\right)} \Theta_{\left(i, t^{\prime \prime}\right)}=0$. Then the equations of system (1) and the degree $(n+1) p d+j$ equations

$$
M_{1}^{(i, j ; t)}=0, \ldots, M_{n(n-i+1)+p(p-i-1)-j+1)}^{(i, j ; t)}=0
$$

intersect transversally at any of their common solutions in $\mathbb{T}_{i} \backslash D_{\left(i, j ; t, t^{\prime}, t^{\prime \prime}\right)}$. They define $\mathfrak{B}_{(i, j)} \backslash D_{\left(i, j ; t, t^{\prime}, t^{\prime \prime}\right)}$ in $\mathbb{T}_{i} \backslash D_{\left(i, j ; t, t^{\prime}, t^{\prime \prime}\right)}$. Moreover, for suitably chosen vectors $\left(t, t^{\prime}, t^{\prime \prime}\right) \in \mathbb{N}^{j-1} \times \mathbb{N}^{n-i} \times \mathbb{N}^{p}$ the union of the sets $\mathbb{T}_{i} \backslash D_{\left(i, j ; t, t^{\prime}, t^{\prime \prime}\right)}$ covers $\mathfrak{B}_{(i, j)} \cap$ 
$H_{i} \backslash \mathfrak{B}_{(i, j-1)}$. There exist $\left(\begin{array}{c}n(n-i+1)+p(p-i-1) \\ j-1\end{array}\right)\left(\begin{array}{c}n \\ n-i\end{array}\right)\left(\begin{array}{c}n-i \\ p\end{array}\right)$ such choices for the vector $\left(t, t^{\prime}, t^{\prime \prime}\right) \in \mathbb{N}^{j-1} \times \mathbb{N}^{n-i} \times \mathbb{N}^{p}$.

Now let $1 \leq j \leq n-p, a_{0} \in \mathbb{A}^{j}$ with $a_{0} \neq 0, a_{*} \in \mathbb{A}^{j \times(n(p+1))}$ generic and $a:=\left[a_{0}^{T}, a_{*}\right]$. Let $\sigma \in \operatorname{Sym}(n)$. For the sake of simplicity of exposition we suppose that $\sigma$ is the identity permutation. Furthermore, let $T_{a}^{(i, \sigma, j)}$ be the polynomial $(((n+1) p+j) \times n(p+1))$-matrix whose first $(n+1) p$ rows constitute the Jacobian of the system (2) of Section 3 and whose remaining $j$ rows are built as in Section 2 in order to define the $(n-p-j+1)$ th dual polar variety of $H_{i, \sigma}$ associated with the linear space $\bar{K}(a)$. Then $\mathcal{B}_{(i, \sigma, j)}$ is the Zariski closure in $\mathbb{F}_{i}$ of the set of all points $(x, b, \lambda, \vartheta) \in H_{i, \sigma}$ where $T_{a}^{(i, \sigma, j)}(x, b, \lambda, \vartheta)$ does not have full rank.

By $\widetilde{T}_{a}^{(i, \sigma, j)}$ we denote the polynomial $(((n+1) p+j-1) \times n(p+1))$-matrix consisting of all rows of $T_{a}^{(i, \sigma, j)}$ except the last one.

Observe again, that the small bipolar varieties $\mathcal{B}_{(i, \sigma, j)}$ of $S$ form a chain of equidimensional varieties

$$
\overline{H_{i, \sigma}} \supsetneqq \mathcal{B}_{(i, \sigma, n-p)} \supset \cdots \supset \mathcal{B}_{(i, \sigma, 1)} .
$$

The variety $\mathcal{B}_{(i, \sigma, 1)}$ is empty or zero-dimensional. If $\mathcal{B}_{(i, \sigma, 1)}$ is nonempty, then the chain is strictly decreasing. We define $\mathcal{B}_{(i, \sigma, 0)}:=\emptyset$.

For $t \in \mathbb{N}^{j-1}$ with $t:=\left(t_{1}, \ldots, t_{j-1}\right)$ and

$$
(n+1) p<t_{1}<\cdots<t_{j-1} \leq n(p+1)
$$

we denote by $m_{(i, \sigma, j ; t)}$ the $((n+1) p+j-1)$-minor of $\widetilde{T}_{a}^{(i, \sigma, j)}$ which corresponds to the first $(n+1) p$ columns and the columns numbered $t_{1}, \ldots, t_{j-1}$ of $\widetilde{T}_{a}^{(i, \sigma, j)}$. Moreover, for

$$
(n+1) p<k_{1}<\cdots<k_{n-p-j+1} \leq n(p+1)
$$

different from $t_{1}, \ldots, t_{j-1}$ and $1 \leq h \leq n-p-j+1$ we denote by $M_{h}^{(i, \sigma, j ; t)}$ the $((n+1) p+j)$-minor of $T_{a}^{(i, \sigma, j)}$ which corresponds to the first $(n+1) p$ columns of $T_{a}^{(i, \sigma, j)}$ and the columns numbered $t_{1}, \ldots, t_{j-1}$ and $k_{h}$. Observe $\operatorname{deg} M_{h}^{(i, \sigma, j ; t)} \leq$ $(n+1) p d$.

Observe that there exist $\left(\begin{array}{c}n-p \\ j-1\end{array}\right)$ choices of vectors $t \in \mathbb{N}^{j-1}$.

In the same way as in the case of Proposition 6. now one proves the following statement.

Proposition 7. Let notation be as before and let $t \in \mathbb{N}^{j-1}$ be a suitable vector. Denote by $D_{(i, \sigma, j ; t)}$ the closed subvariety of $\mathbb{F}_{i}$ defined by the equation $m_{(i, \sigma, j ; t)}=0$. Then the equations of system (2) and the degree $(n+1) p d$ equations

$$
M_{1}^{(i, \sigma, j ; t)}=0, \ldots, M_{n-p-j+1}^{(i, \sigma, j ; t)}=0
$$

intersect transversally at any of their common solutions in $\mathbb{F}_{i} \backslash D_{(i, \sigma, j ; t)}$. They define $\mathcal{B}_{(i, \sigma, j)} \backslash D_{(i, \sigma, j ; t)}$ in $\mathbb{F}_{i} \backslash D_{(i, \sigma, j ; t)}$. Moreover, for suitably chosen vectors $t \in \mathbb{N}^{j-1}$ the union of the sets $\mathbb{F}_{i} \backslash D_{(i, \sigma, j ; t)}$ covers $\mathcal{B}_{(i, \sigma, j)} \cap H_{i, \sigma} \backslash \mathcal{B}_{(i, \sigma, j-1)}$. There exist $\left(\begin{array}{c}n-p \\ j-1\end{array}\right)$ possible choices of vectors $t \in \mathbb{N}^{j-1}$. 
4.2. Degrees of bipolar varieties. We denote by $\operatorname{deg} \mathfrak{B}_{(i, j)}$ and $\operatorname{deg} \mathcal{B}_{(i, \sigma, j)}$ the geometric degrees of the respective bipolar varieties in their ambient spaces $\mathbb{T}_{i}$ and $\mathbb{F}_{i}$ (see [26] for a definition and properties of the geometric degree of a subvariety of an affine space).

Observe that $\operatorname{deg} \mathfrak{B}_{(i, j)}$ remains invariant under linear transformations of the coordinates $X_{1}, \ldots, X_{n}$ by unitary complex matrices.

From [8, Lemma 1] and [5, Theorem 3] we deduce that for $1 \leq j \leq n-p$,

$$
\operatorname{deg} \mathcal{B}_{(i, \sigma, j)} \leq \operatorname{deg} \mathfrak{B}_{(i, n(n-i))+p(p-i)+j)}
$$

holds.

Suppose that $S$ contains a regular real point $x$. Then there exists a permutation $\sigma \in \operatorname{Sym}(n)$ with $\Delta_{\sigma}(x) \neq 0$. From Proposition 3 we deduce that $\left(H_{i, \sigma}\right)_{\mathbb{R}}$ is nonempty. This implies that $H_{i, \sigma}$ is given by a reduced regular sequence of polynomials, namely the polynomials in system (2). Moreover, the real variety $\left(H_{i, \sigma}\right)_{\mathbb{R}}$ is smooth. Therefore we may apply [3], 44. Proposition 2] to conclude that $\mathcal{B}_{(i, \sigma, j)_{\mathbb{R}}}$ contains for each connected component of $\left(H_{i, \sigma}\right)_{\mathbb{R}}$ at least one point. This implies

$$
1 \leq \operatorname{deg} \mathcal{B}_{(i, \sigma, 1)} \leq \operatorname{deg} \mathfrak{B}_{(i, n(n-i))+p(p-i)+1)} .
$$

For $1 \leq r \leq p, 1 \leq l \leq n$ and $\sigma \in \operatorname{Sym}(n)$ we are going to analyze in the following closed subvarieties $S_{(r, l)}^{(i)}$ and $S_{(r, l)}^{(i, \sigma)}$ of the affine subspaces $\mathbb{T}_{i}$ and $\mathbb{F}_{i}$, respectively. For this purpose we consider the lexicographical order $<$ of the set of all pairs $(r, l)$ with $1 \leq r \leq p, \quad 1 \leq l \leq n$.

Let $S_{(r, l)}^{(i)}$ be the Zariski closure of the locally closed subset of $\mathbb{T}_{i}$ defined by the conditions

$$
\begin{gathered}
F_{1}(X)=\cdots=F_{p}(X)=0 \\
\sum_{1 \leq s \leq p} \Lambda_{r^{\prime}, s} \frac{\partial F_{s}}{\partial X_{l^{\prime}}}+\sum_{1 \leq k \leq n-i} B_{k, l^{\prime}} \Theta_{k, r}=0, \\
1 \leq r^{\prime} \leq p, \quad 1 \leq l^{\prime} \leq n, \quad\left(r^{\prime}, l^{\prime}\right) \leq(r, l), \text { and } \\
\text { rk } B=n-i, \text { rk } \Theta=p \text { and rk } J(F)=p .
\end{gathered}
$$

Observe that the particular structure of the Jacobian of the equations of system (44) implies that the corresponding polynomials form a reduced regular sequence at any of their common zeros outside of the closed locus given by the conditions

$$
\text { rk } B<n-i, \operatorname{rk} \Theta<p \text { or } \operatorname{rk} J(F)<p .
$$

Furthermore, let $S_{(r, l)}^{(i, \sigma)}$ be the locally closed subset of $\mathbb{F}_{i}$ defined by the conditions

$$
\begin{gathered}
F_{1}(X)=\cdots=F_{p}(X)=0, \\
\sum_{1 \leq s \leq p} \Lambda_{r^{\prime}, s} \frac{\partial F_{s}}{\partial X_{l^{\prime}}}+\delta_{r^{\prime}, l^{\prime}}=0, \quad 1 \leq r^{\prime} \leq r, \quad 1 \leq l^{\prime} \leq p, \quad\left(r^{\prime}, l^{\prime}\right) \leq(r, l),
\end{gathered}
$$

5) $\sum_{1 \leq s \leq p} \Lambda_{r^{\prime}, s} \frac{\partial F_{s}}{\partial X_{l^{\prime}}}+\Theta_{l^{\prime}, r^{\prime}}=0, \quad 1 \leq r^{\prime} \leq r, p<l^{\prime} \leq n-i, \quad\left(r^{\prime}, l^{\prime}\right) \leq(r, l)$,

$$
\sum_{1 \leq s \leq p} \Lambda_{r^{\prime}, s} \frac{\partial F_{s}}{\partial X_{l^{\prime}}}+B_{r^{\prime}, l^{\prime}}=0, \quad 1 \leq r^{\prime} \leq r, \quad n-i<l \leq n, \quad\left(r^{\prime}, l^{\prime}\right) \leq(r, l),
$$

and $\Delta_{\sigma}(X) \neq 0$. 
Again the particular structure of the Jacobian of the equations of system (15) implies that the corresponding polynomials form a reduced regular sequence at any of their common zeros outside of the closed locus given by the condition $\Delta_{\sigma}(X)=0$.

In conclusion, the polynomials of systems (11) and (2) form strongly reduced regular sequences at any of their common zeros outside of the corresponding closed loci.

For the next statement recall that the degree of the polynomials $F_{1}, \ldots, F_{p}$ is bounded by $d$ (see Section 2).

Proposition 8. Let $1 \leq r \leq p$ and $1 \leq l \leq n$. Then we have the extrinsic estimate

$$
\operatorname{deg} S_{(r, l)}^{(i)} \leq d^{p(n+1)}=d^{O\left(n^{2}\right)} .
$$

Proof. Without loss of generality we may suppose $d \geq 2$. Then we deduce from the Bézout Inequality $([18,26,45])$ that the closed subvariety of $\mathbb{T}_{i}$ defined by the equations of system (41) is of degree at most $d^{p(n+1)}=d^{O\left(n^{2}\right)}$.

In fact, this bound is too coarse, because refined methods, based on the multihomogeneous Bézout Inequality of [34, yield an estimate $\operatorname{deg} S_{(r, l)}^{(i)}=\left(n^{n} d\right)^{O(n)}$ which is sharper for $d \geq n$. This improvement will not be very relevant in Section 5 where the degree of $S_{(r, l)}^{(i)}$ plays a key role in complexity estimates. More important will be the estimate $\operatorname{deg} S_{(r, l)}^{(i, \sigma)}=(n d)^{O(n)}, \sigma \in \operatorname{Sym}(n)$, we are going to derive now.

Lemma 9. Let $1 \leq r \leq p, \Lambda^{(r)}:=\left[\Lambda_{r^{\prime}, s}\right]_{\substack{1 \leq r^{\prime} \leq r \\ 1 \leq s \leq p}}$ and $\Delta:=\operatorname{det}\left[\frac{\partial F_{s}}{\partial X_{l^{\prime}}}\right]_{1 \leq s, l^{\prime} \leq p}$. Then the Zariski closure of the locally closed subvariety $\mathfrak{S}_{r}$ of $\mathbb{A}^{n} \times \mathbb{A}^{r \times p}$ defined by the conditions

$$
\begin{gathered}
F_{1}(X)=\cdots=F_{p}(X)=0, \\
\sum_{1 \leq s \leq p} \Lambda_{r^{\prime}, s} \frac{\partial F_{s}}{\partial X_{l^{\prime}}}+\delta_{r^{\prime}, l^{\prime}}=0,1 \leq r^{\prime} \leq r, \quad 1 \leq l^{\prime} \leq p \\
\Delta \neq 0
\end{gathered}
$$

is empty or equidimensional of dimension $n-p$. Its geometric degree is bounded by $(2 n d)^{n}$. The polynomials of system (6) form a reduced regular sequence in $\mathbb{Q}\left[X, \Lambda^{(r)}\right]_{\Delta}$.

Proof. From ([6) one deduces easily that for a point $(x, \lambda) \in \mathfrak{S}_{r}$ with $\Delta(x) \neq 0$ the matrix $\left[\frac{\partial F_{s}}{\partial X_{l^{\prime}}}\right]_{\substack{1 \leq s \leq r \\ 1 \leq l^{\prime} \leq p}}$ has maximal rank. This implies that the Jacobian of (6) has full rank at $(x, \lambda)$. Hence the variety $\mathfrak{S}_{r}$ is smooth and of dimension $n-p$ at $(x, \lambda)$. Thus $\mathfrak{S}_{r}$ is empty or equidimensional of dimension $n-p$. Moreover, the polynomials of system (6) form a reduced regular sequence in $\mathbb{Q}\left[X, \Lambda^{(r)}\right]_{\Delta}$.

Observe that for $x \in S$ with $\Delta(x) \neq 0$ there exists exactly one point $\lambda \in \mathbb{A}^{r \times p}$ such that $(x, \lambda)$ belongs to $\mathfrak{S}_{r}$. Thus $\mathfrak{S}_{r} \cap\left(S_{\Delta} \times \mathbb{A}^{r \times p}\right)$ is the graph of a rational map $\varphi: S \rightarrow \mathbb{A}^{r \times p}$ which is everywhere defined on $S_{\Delta}$. By Cramer's rule each component of this rational map may be described by a rational expression whose numerator is a polynomial of $\mathbb{Q}[X]$ of degree at most $p d$ and whose denominator is $\Delta$.

Let $K_{1}, \ldots, K_{n-p}$ be generic affine linear polynomials of $\mathbb{Q}\left[X, \Lambda^{(r)}\right]$. Then we have 
$\operatorname{deg} \mathfrak{S}_{r}=\#\left(\mathfrak{S}_{r} \cap\left\{K_{1}=0, \ldots, K_{n-p}=0\right\}\right)$, where \# denotes the cardinality of the corresponding set. Without loss of cardinality we may suppose that $\mathfrak{S}_{r} \cap\left\{K_{1}=0, \ldots, K_{n-p}=0\right\}$ is contained in $\mathbb{A}_{\Delta}^{n} \times \mathbb{A}^{r \times n}$ (see [26, Remark 2]). Replacing in $K_{1}=0, \ldots, K_{n-p}=0$ each indeterminate $\Lambda_{r^{\prime}, s}, 1 \leq r^{\prime} \leq r, 1 \leq s \leq p$ by the given rational expression for the corresponding coordinate of $\varphi$ and cleaning the denominator $\Delta$ we obtain together with $F_{1}, \ldots, F_{p}$ a system of $n$ polynomials of $\mathbb{Q}[X]$ having degree at most $2 p d$ which in $\mathbb{A}_{\Delta}^{n}$ defines a set of the same cardinality as $\mathfrak{S}_{r} \cap\left\{K_{1}=0, \ldots, K_{n-p}=0\right\}$. From the Bézout Inequality we therefore deduce

$$
\operatorname{deg} \mathfrak{S}_{r} \leq(2 p d)^{n} \leq(2 n d)^{n} .
$$

Proposition 10. Let $1 \leq r \leq p$ and $1 \leq l \leq n$. Then we have the estimate

$$
\operatorname{deg} S_{(r, l)}^{(i, \sigma)} \leq\left(2 n d^{3}\right)^{n} .
$$

Proof. Without loss of generality we may suppose that $\sigma \in \operatorname{Sym}(n)$ is the identity permutation. Then we have, with the notation of the previous lemma, $\Delta=\Delta_{\sigma}$.

We consider $\mathfrak{S}_{r-1} \times \mathbb{A}^{p}$ and $\mathfrak{S}_{r}$ as closed subvarieties of $\mathbb{A}^{n} \times \mathbb{A}^{r \times p}$ with the convention $\mathfrak{S}_{0}:=\overline{S_{\Delta}}$. Let $V_{(r, l)}$ be the Zariski closure of the locally closed subvariety of $\mathbb{A}^{n} \times \mathbb{A}^{r \times p}$ defined by the conditions

$$
\begin{gathered}
F_{1}(X)=\cdots=F_{p}(X)=0, \\
\sum_{1 \leq s \leq p} \Lambda_{r^{\prime}, s} \frac{\partial F_{s}}{\partial X_{l^{\prime}}}+\delta_{r^{\prime}, l^{\prime}}=0, \quad 1 \leq r^{\prime} \leq r, \quad 1 \leq l^{\prime} \leq p,\left(r^{\prime}, l^{\prime}\right) \leq(r, l), \\
\text { and } \quad \Delta \neq 0
\end{gathered}
$$

Observe that $V_{(r, l)}$ is the intersection of $\mathfrak{S}_{r-1} \times \mathbb{A}^{p}$ with the subvariety of $\mathbb{A}^{n} \times \mathbb{A}^{r \times p}$ defined by the equations

$$
\sum_{1 \leq s \leq p} \Lambda_{r, s} \frac{\partial F_{s}}{\partial X_{l^{\prime}}}+\delta_{r, l^{\prime}}=0, \quad 1 \leq l^{\prime} \leq \min \{l, p\} .
$$

From the Bézout Inequality and Lemma 9 we infer

$$
\operatorname{deg} V_{(r, l)} \leq d^{p} \operatorname{deg} \mathfrak{S}_{r} \leq\left(n d^{2}\right)^{n} .
$$

By Lemma 9 the variety $\mathfrak{S}_{r-1}$ is empty or of dimension $n-p$. Since $\mathfrak{S}_{r-1} \times \mathbb{A}^{p}$ contains $V_{(r, l)}$ we conclude $\operatorname{dim} V_{(r, l)} \leq n$. Observe now that system (5) describes the graph of a rational map with domain $\left(V_{(r, l)}\right)_{\Delta}$. The polynomials which constitute this map have degree at most $d$. In the same way as in the proof of Lemma 9 , the Bézout Inequality implies that system (15) describes a locally closed algebraic subvariety of $\mathbb{F}_{i}$ whose Zariski closure is of degree at most $d^{n} \operatorname{deg} V_{(r, l)} \leq\left(2 n d^{3}\right)^{n}$. Since the irreducible components of $S_{(r, l)}^{(i, \sigma)}$ are irreducible components of this variety we conclude that $\operatorname{deg} S_{(r, l)}^{(i, \sigma)} \leq\left(2 n d^{3}\right)^{n}$ holds.

Observation 11. Let $1 \leq r \leq p$ and $1 \leq l \leq n$. Then the estimate of Proposition 8 can be improved to $\operatorname{deg} S_{(r, l)}^{(i)}=\left(n^{n} d\right)^{O(n)}$.

Proof (Sketch). Let us first suppose $p \leq l \leq n$ and let $Y:=\left[Y_{r^{\prime \prime}, s}\right]_{1 \leq r^{\prime \prime}, s \leq p}$ be a matrix of new indeterminates. Consider again $\Delta:=\operatorname{det}\left[\frac{\partial F_{s}}{\partial X_{l}}\right]_{1 \leq s, l \leq p}$. We show 
$\operatorname{deg} \overline{\left(S_{(r, l)}^{i}\right)_{\Delta}}=(n d)^{O(n)}$. For this purpose we add to the equations of (4) the equations

$$
\sum_{1 \leq s \leq p} Y_{r^{\prime \prime}, s} \frac{\partial F_{s}}{\partial X_{l^{\prime \prime}}}=\delta_{r^{\prime \prime}, l^{\prime \prime}}, \quad 1 \leq r^{\prime \prime}, l^{\prime \prime} \leq p .
$$

In this way we obtain a closed subvariety $W$ of $\mathbb{T}_{i} \times \mathbb{A}^{p \times p}$. Taking into account the assumption $p \leq l \leq n$, one sees easily that the Jacobian of the system composed by (4) and (7) has at any point of $W$ full rank. Therefore, $W$ is empty or an equidimensional, smooth variety. The system composed by (4) and (7) contains five types of variables, namely the ones contained in $B, \Lambda, \Theta$ and $Y$ (which occur linearly) and the variables $X_{1}, \ldots, X_{n}$ (which occur in degree at most $d$ ). Intersecting $W$ with $\operatorname{dim} W$ many affine hyperplanes of $\mathbb{T}_{i} \times \mathbb{A}^{p \times p}$ given by generic affine linear polynomials of $\mathbb{Q}[X, B, \Lambda, \Theta, Y]$, we deduce from the dehomogenized version of the multi-homogeneous Bézout Inequality of [34] or from [15. Corollary 1.12] the estimate $\operatorname{deg} W=\left(n^{n} d\right)^{O(n)}$.

Let $\pi: \mathbb{T}_{i} \times \mathbb{A}^{p \times p} \rightarrow \mathbb{T}_{i}$ be the canonical projection from $\mathbb{T}_{i} \times \mathbb{A}^{p \times p}$ onto $\mathbb{T}_{i}$ and observe that $\overline{\pi(W)}$ is birationally equivalent to $W$ and hence empty or an equidimensional subvariety of $\mathbb{T}_{i}$. Since the irreducible components of $\overline{\left(S_{(r, l)}^{(i)}\right)_{\Delta}}$ are irreducible components of $\overline{\pi(W)}$ we infer from [26, Lemma 2] and its proof the estimate

$$
\operatorname{deg} \overline{\left(S_{(r, l)}^{(i)}\right)_{\Delta}} \leq \operatorname{deg} W=\left(n^{n} d\right)^{O(n)}
$$

In a similar way one sees $\operatorname{deg} \overline{\left(S_{(r, l)}^{(i)}\right)_{\Delta_{\sigma}}}=\left(n^{n} d\right)^{O(n)}$ for any permutation $\sigma \in$ Sym $(n)$. Since there exist $\left(\begin{array}{l}n \\ p\end{array}\right)$ many $p$-minors of $J(F)$ we conclude $\operatorname{deg} S_{(r, l)}^{(i)}=$ $\left(\begin{array}{l}n \\ p\end{array}\right)\left(n^{n} d\right)^{O(n)}=\left(n^{n} d\right)^{O(n)}$. Finally, we consider $S_{(r, l)}^{(i)}$ for $1 \leq r \leq p$ and arbitrary $1 \leq l \leq n$. From (41) we conclude that the irreducible components of $S_{(r, l)}^{(i)}$ are irreducible components of the intersection of $S_{(r-1, n)}^{(i)}$ with $l \leq n$ hypersurfaces of $\mathbb{T}_{i}$ of degree at most $\max \{2, d\}$. Since we have by our previous argumentation, $\operatorname{deg} S_{(r-1, l)}^{(i)}=\left(n^{n} d\right)^{O(n)}$, we deduce from the Bézout Inequality $\operatorname{deg} S_{(r, l)}^{(i)}=\left(n^{n} d\right)^{O(n)}$.

Let $1 \leq i \leq n-p$. We proceed now to derive two extrinsic estimates for the degrees of the bipolar varieties $\mathfrak{B}_{(i, j)}, 1 \leq j \leq n(n-i+1)+p(p-i+1)$, and $\mathcal{B}_{(i, \sigma, j)}, \sigma \in \operatorname{Sym}(n), 1 \leq j \leq n-p$.

Proposition 12. For $1 \leq j \leq n(n-i+1)+p(p-i-1)$ one has the extrinsic estimate $\operatorname{deg} \mathfrak{B}_{(i, j)}=(n d)^{O\left(n^{2}\right)}$. In particular, for $n(n-i)+p(p-i)<j \leq$ $n(n-i+1)+p(p-i-1)$ one has the estimate $\operatorname{deg} \mathfrak{B}_{(i, j)}=\left(n^{n} d\right)^{O(n)}$.

Proof. From Proposition 8 we deduce that the degree of $\overline{H_{i}}$ in $\mathbb{T}_{i}$ is bounded by $d^{p(n+1)}=d^{O\left(n^{2}\right)}$. Observation 11 yields the estimate $\operatorname{deg} \overline{H_{i}}=\left(n^{n} d\right)^{O(n)}$. On the other hand, we deduce from Proposition 6 and the Bézout Inequality that $\operatorname{deg} \mathfrak{B}_{(i, j)}$ is bounded by

$$
\operatorname{deg} \overline{H_{i}}\left(\begin{array}{c}
n(n-i+1)+p(p-i-1) \\
j-1
\end{array}\right)\left(\begin{array}{c}
n \\
n-i
\end{array}\right)\left(\begin{array}{c}
n-i \\
p
\end{array}\right)((n+1) p d+j)^{n(n-i+1)+p(p-i-1)-j+1} .
$$


This implies for $1 \leq j \leq n(n-i+1)+p(p-i-1)$ the general estimate deg $\mathfrak{B}_{(i, j)}=$ $(n d)^{O\left(n^{2}\right)}$ and for $n(n-i)+p(p-i) \leq j \leq n(n-i+1)+p(p-i-1)$ the particular estimate $\operatorname{deg} \mathfrak{B}_{(i, j)}=\left(n^{n} d\right)^{O(n)}$.

Proposition 13. The extrinsic estimate $\operatorname{deg} \mathcal{B}_{(i, \sigma, j)}=(n d)^{O(n)}$ is valid for any $\sigma \in \operatorname{Sym}(n)$ and $1 \leq j \leq n-p$.

Proof. From Proposition 10 we deduce that the degree of $H_{i, \sigma}$ is bounded by $\left(n d^{3}\right)^{n}$. Moreover, Proposition 7 and the Bézout Inequality imply that $\operatorname{deg} \mathcal{B}_{(i, \sigma, j)}$ is bounded by $\operatorname{deg} H_{i, \sigma}\left(\begin{array}{c}n-p \\ j-1\end{array}\right)((n+1) p d+j)^{n-p-j+1}=(n d)^{O(n)}$.

We associate now with $1 \leq i \leq n-p, \sigma \in \operatorname{Sym}(n)$ and the polynomial equation system $F_{1}=\cdots=F_{p}=0$ the following discrete parameters, namely

$$
\begin{array}{r}
\delta_{i}:=\max \left\{\max \left\{\operatorname{deg}\left\{F_{1}=0 \cdots=F_{s}=0\right\} \mid 1 \leq s \leq p\right\},\right. \\
\max \left\{\operatorname{deg} S_{(r, l)}^{(i)} \mid 1 \leq r \leq p, 1 \leq l \leq n\right\}, \\
\left.\max \left\{\operatorname{deg} \mathfrak{B}_{i, n(n-i)+p(p-i)+j} \mid 1 \leq j \leq n-p\right\}\right\}
\end{array}
$$

and

$$
\begin{array}{r}
\delta_{i, \sigma}:=\max \left\{\max \left\{\operatorname{deg}\left\{F_{1}=0 \cdots=F_{s}=0\right\} \mid 1 \leq s \leq p\right\},\right. \\
\max \left\{\operatorname{deg} S_{(r, l)}^{(i, \sigma)} \mid 1 \leq r \leq p, 1 \leq l \leq n\right\}, \\
\left.\max \left\{\operatorname{deg} \mathcal{B}_{(i, \sigma, j)} \mid 1 \leq j \leq n-p\right\}\right\} .
\end{array}
$$

Adapting the terminology of [8, Section 4.2] and taking into account that for $1 \leq j \leq n-p$ the degree of $\mathfrak{B}_{(i, n(n-i)+p(p-i)+j)}$ remains invariant under linear transformations of the coordinates $X_{1}, \ldots, X_{n}$ by unitary complex matrices, we call $\delta_{i}$ and $\delta_{i, \sigma}$ the unitary-independent and the unitary-dependent degree of the real interpretation of the equation system $F_{1}=\cdots=F_{p}=0$ associated with $i$ and $\sigma$.

Observe that (3) and the Bézout Inequality imply

$$
\delta_{i, \sigma} \leq \delta_{i} \quad \text { for any } \sigma \in \operatorname{Sym}(n) .
$$

From Propositions 10, 12, 13, Observation 11, and the Bézout Inequality we deduce the following extrinsic estimates:

$$
\delta_{i}=\left(n^{n} d\right)^{O(n)}
$$

and

$$
\delta_{i, \sigma}=(n d)^{O(n)}
$$

(compare for the case $p:=1$ the estimates (16) and (17) given in [8, Section 4.2]).

For the rest of the paper we fix a family $\left\{\sigma_{1}, \ldots, \sigma_{\left(\begin{array}{c}n \\ p\end{array}\right)}\right\}$ of permutations from Sym $(n)$ such that for any choice $1 \leq k_{1}<\cdots<k_{p} \leq n$ there exists an index $1 \leq k \leq\left(\begin{array}{l}n \\ p\end{array}\right)$ with $\sigma_{k}(1)=k_{1}, \ldots, \sigma_{k}(p)=k_{p}$.

Let $\widetilde{\delta}_{i}:=\sum_{1 \leq k \leq\left(\begin{array}{c}n \\ p\end{array}\right)} \delta_{i, \sigma_{k}}$. From (9) we deduce

$$
\widetilde{\delta}_{i}=(n d)^{O(n)} .
$$

Observe finally that

$$
\widetilde{\delta}_{i} \leq\left(\begin{array}{c}
n \\
p
\end{array}\right) \delta_{i}
$$

holds. 


\section{Real RoOt Finding FOR $F_{1}=0, \ldots, F_{p}=0$}

We are going to present a discrete family of efficient non-uniform (or alternatively uniform probabilistic) procedures $\Pi_{i}, 1 \leq i \leq n-p$, which satisfy the following specifications. Let $Z$ be a new indeterminate.

\section{Input:}

An essentially division-free arithmetic circuit $\beta$ in $\mathbb{Q}[X]$ of size $L$ and non-scalar depth $\ell$ having $p$ output nodes.

\section{Input specification:}

The circuit $\beta$ represents by its output nodes $p$ polynomials $F_{1}, \ldots, F_{p} \in \mathbb{Q}[X]$ of maximal degree $d$. These polynomials form a strongly reduced regular sequence in $\mathbb{Q}[X]$.

\section{Output:}

The procedure $\Pi_{i}$ accepts the input $\beta$ if $S:=\left\{F_{1}=0, \ldots, F_{p}=0\right\}$ contains a smooth real point. If this is the case, the procedure returns a circuit representation of the coefficients of $n+1$ polynomials $P, G_{1}, \ldots, G_{n} \in \mathbb{Q}[Z]$ satisfying for $G:=$ $\left(G_{1}, \ldots, G_{n}\right)$ the following

\section{Output specification:}

- $\mathrm{P}$ is monic and separable,

- $\operatorname{deg} G<\operatorname{deg} P \leq \widetilde{\delta}_{i}$, where $\operatorname{deg} G:=\max \left\{\operatorname{deg} G_{1}, \ldots, \operatorname{deg} G_{n}\right\}$,

- the zero-dimensional affine variety

$$
\left\{G(z) \mid z \in \mathbb{A}^{1}, P(z)=0\right\}
$$

contains a smooth real point of each generically smooth connected component of $S_{\mathbb{R}}$. In order to represent these sample points, an encoding "à la Thom" of the real zeros of the polynomial $P$ is returned (see e.g. [14] for this kind of encoding).

We say that $\Pi_{i}$ solves the real root finding problem for $F_{1}=0, \ldots, F_{p}=0$.

We now fix $1 \leq i \leq n-p$ and $\sigma \in \operatorname{Sym}(n)$. Without loss of generality we may suppose that $\sigma$ is the identity permutation. From Proposition 3 we deduce that the equations of system (2) intersect transversally at any of their real solutions. Moreover, the polynomials of (2) form in $\mathbb{Q}[X, \mathbf{B}, \Lambda, \boldsymbol{\Theta}]$ a strongly reduced regular sequence (see Section 4.2).

Denote by $V^{\sigma}:=H_{i, \sigma}$ the closed algebraic subvariety of $\mathbb{F}_{i}$ consisting of the common complex solutions of the polynomial equation system (2) and let $V_{\mathbb{R}}^{\sigma}:=$ $V^{\sigma} \cap\left(\mathbb{F}_{i}\right)_{\mathbb{R}}$ be the real trace of $V^{\sigma}$ in $\mathbb{F}_{i}$. Thus $V_{\mathbb{R}}^{\sigma}$ consists of all real solutions of (2) and is therefore closed in the Euclidean topology. Moreover, from Proposition 3 we conclude that $V^{\sigma}$ and $V_{\mathbb{R}}^{\sigma}$ are empty or smooth of dimension $n-p$ and that the real variety $V_{\mathbb{R}}^{\sigma}$ is non-empty if and only if $S$ contains a real point $x$ with $\Delta_{\sigma}(x) \neq 0$. More precisely, for any connected component $C$ of $S_{\mathbb{R}}$, where $\Delta_{\sigma}$ do not vanish identically, there exists a point $(x, b, \lambda, \vartheta)$ of $V_{\mathbb{R}}^{\sigma}$ with $x \in C, \Delta_{\sigma}(x) \neq 0$ and $(b, \lambda, \vartheta) \in \mathbb{A}_{\mathbb{R}}^{p \times i} \times \mathbb{A}_{\mathbb{R}}^{p \times p} \times \mathbb{A}_{\mathbb{R}}^{(n-p-i) \times p}$.

Therefore, a set of algebraic sample points for the connected components of $V_{\mathbb{R}}^{\sigma}$ gives rise to a set of algebraic sample points for the connected components of $S_{\mathbb{R}}$ where $\Delta_{\sigma}$ does not vanish identically.

Suppose now that $S$ contains a real point $x$ with $\Delta_{\sigma}(x) \neq 0$. Then the real variety $V_{\mathbb{R}}^{\sigma}$ is smooth and equidimensional of dimension $n-p$. For $1 \leq j \leq n-p$ we infer from [4], Proposition 2 that the real bipolar variety $\left(\mathcal{B}_{(i, \sigma, j)}\right)_{\mathbb{R}}$ (and hence the complex variety $\mathcal{B}_{(i, \sigma, j)}$ ) contains at least one point of each connected component 
of $V_{\mathbb{R}}^{\sigma}$. Thus $\mathcal{B}_{(i, \sigma, j)}$ and $\left(\mathcal{B}_{(i, \sigma, j)}\right)_{\mathbb{R}}$ are equidimensional of dimension $j-1$. From Proposition 7 we conclude that for $1 \leq j \leq n-p$ the algebraic variety $\mathcal{B}_{(i, \sigma, j)} \backslash$ $\mathcal{B}_{(i, \sigma, j-1)}$ is locally definable by reduced regular sequences. In particular, $\mathcal{B}_{(i, \sigma, 1)}$ is zero-dimensional and contains for each connected component of $V_{\mathbb{R}}^{\sigma}$ an algebraic sample point. The algorithm $\Pi_{i}$ proceeds now by deciding for each $1 \leq k \leq\left(\begin{array}{l}n \\ p\end{array}\right)$ whether $\mathcal{B}_{\left(i, \sigma_{k}, 1\right)}$ contains real algebraic points, and, if it is the case, by computing them. The algorithm infers from these data whether $S$ contains smooth real points. If the answer is positive, the set of data furnish also a finite set of smooth real algebraic sample points for the generically smooth connected components of $S_{\mathbb{R}}$.

At the beginning, the procedure $\Pi_{i}$ generates for each $1 \leq k \leq\left(\begin{array}{l}n \\ p\end{array}\right)$ from the input circuit $\beta$ a new division-free circuit $\beta_{\sigma_{k}}$ of size $O(L+n p)$ and non-scalar depth $\ell+O(1)$ that represents by its output nodes the polynomials of $\mathbb{Q}[X, \mathbf{B}, \Lambda, \boldsymbol{\Theta}]$ which define as in Section 3 the variety $H_{i, \sigma_{k}}$. For the sake of simplicity we fix $\sigma:=\sigma_{k}$ and suppose that $\sigma$ is the identity permutation of Sym $(n)$. Taking the circuit $\beta_{\sigma}$ as input, the procedure $\Pi_{i}$ follows now the pattern of the (non-uniform or probabilistic) procedure described in [3, Theorem 11] and 4, Theorem 13] in order to decide whether $V_{\mathbb{R}}^{\sigma}$ is empty.

If $V_{\mathbb{R}}^{\sigma}$ is empty then the procedure $\Pi_{i}$ returns the answer that $\Delta_{\sigma}$ vanishes identically on any connected component of $S_{\mathbb{R}}$.

Suppose that $V_{\mathbb{R}}^{\sigma}$ is not empty. Then the procedure $\Pi_{i}$ returns the coefficients of $n(p+1)+1$ polynomials $P^{\sigma}, G_{1}^{\sigma}, \ldots, G_{n}^{\sigma}, G_{n+1}^{\sigma}, \ldots, G_{n(p+1)}^{\sigma} \in \mathbb{Q}[Z]$ satisfying for $G^{\sigma}:=\left(G_{1}^{\sigma}, \ldots, G_{n(p+1)}^{\sigma}\right)$ the following conditions:

- $P^{\sigma}$ is monic and separable,

- $\operatorname{deg} G^{\sigma}<\operatorname{deg} P^{\sigma} \leq \operatorname{deg} \mathcal{B}_{(i, \sigma, 1)}$,

- $\mathcal{B}_{(i, \sigma, 1)}=\left\{G^{\sigma}(z) \mid z \in \mathbb{A}^{1}, P^{\sigma}(z)=0\right\}$.

From this representation of the variety $\mathcal{B}_{(i, \sigma, 1)}$ we deduce that for $G_{\sigma}:=\left(G_{1}^{\sigma}, \ldots\right.$, $\left.G_{n}^{\sigma}\right)$ the zero-dimensional variety $\left\{G_{\sigma}(z) \mid z \in \mathbb{A}^{1}, P^{\sigma}(z)=0\right\}$ contains a real algebraic sample point for each connected component of $S_{\mathbb{R}}$ where $\Delta_{\sigma}$ does not vanish identically. The procedure $\Pi_{i}$ now collects this information for any permutation $\sigma_{k}, 1 \leq k \leq\left(\begin{array}{l}n \\ p\end{array}\right)$, in order to construct $n+1$ polynomials $P, G_{1}, \ldots, G_{n} \in \mathbb{Q}[Z]$ which satisfy the output specifications above.

This is done in the following way. The polynomial $P$ is obtained by taking the product of all polynomials $P^{\sigma_{k}}$ with $V_{\mathbb{R}}^{\sigma_{k}} \neq \emptyset, 1 \leq k \leq\left(\begin{array}{l}n \\ p\end{array}\right)$ and making the result squarefree. Then we have $\operatorname{deg} P \leq \widetilde{\delta}_{i}$. From $P$ the polynomials $G_{1}, \ldots, G_{n}$ are easily obtained applying a standard algorithm which goes back to Kronecker (see 23. for details). In this way the procedure $\Pi_{i}$ produces a set of real algebraic sample points for the generically smooth connected components of $S_{\mathbb{R}}$ from $G$ and the encoding "à la Thom" of the real zeros of $P$.

The procedure $\Pi_{i}$ is based on the original paradigm [20,21] of a procedure with intrinsic complexity that solves polynomial equation systems over the complex numbers (see also $17,19,23]$ ).

We are now going to describe succinctly the procedure $\Pi_{i}$ (Propositions 7 and 10 will play here a key role). For this purpose we shall freely refer to terminology, mathematical results and subroutines of [23, where the first streamlined version of the polynomial equation solver [20,21] was presented and implemented as the "Kronecker-algorithm" (compare also [29]). 
In order to simplify the exposition we shall refrain from the presentation of details which ensure only appropriate genericity conditions for the procedure. The following description requires that the reader is acquainted with the details of the Kronecker-algorithm. Although at first glance this description may look intricate, no substantially new idea, which has not been explained before, is introduced.

As before, we consider the identity permutation $\sigma \in \operatorname{Sym}(n)$. Recall that the polynomials of (2) generate the trivial ideal or form a strongly reduced regular sequence in $\mathbb{Q}[X, \mathbf{B}, \Lambda, \boldsymbol{\Theta}]_{\Delta_{\sigma}}$. In this situation the procedure $\Pi_{i}$ applies to system (2) the algorithm "Geometric Solve" of [23] to decide whether $V^{\sigma}=H_{i, \sigma}$ is empty. In this case the information that $V_{\mathbb{R}}^{\sigma}$ does not contain any smooth point is returned. Suppose that this is not the case. Then the algorithm "Geometric Solve" returns a lifting fiber of the variety $V^{\sigma}$.

Next, beginning with $j:=n-p$, the procedure $\Pi_{i}$ decides for any index $1 \leq j \leq$ $n-p$ whether the variety $\mathcal{B}_{(i, \sigma, j)}$ is empty or returns a lifting fiber of it. In case there exists an index $1 \leq j \leq n-p$ with $\mathcal{B}_{(i, \sigma, j)}=\emptyset$, the procedure $\Pi_{i}$ returns the information that $V_{\mathbb{R}}^{\sigma}$ does not contain any smooth point. Suppose that this is not the case.

For $1 \leq j \leq n-p$ we fix a vector $t^{(j)} \in \mathbb{N}^{j-1}$ with $t^{(j)}:=\left(t_{1}^{(j)}, \ldots, t_{j-1}^{(j)}\right)$ and $(n+1) p<t_{1}^{(j)}<\cdots<t_{j-1}^{(j)} \leq n(p+1)$. In the same way as in [8, Section 4.3] one sees that the minor $m_{\left(i, \sigma, j ; t^{(j)}\right)}$ does not vanish identically on any irreducible component of $\mathcal{B}_{(i, \sigma, j)}$.

Following Proposition 7 the equations of system (2) and the equations

$$
M_{1}^{\left(i, \sigma, j, t^{(j)}\right)}=0, \ldots, M_{n-p-j+1}^{\left(i, \sigma, j, t^{(j)}\right)}=0
$$

define the variety $\left(\mathcal{B}_{(i, \sigma, j)}\right)_{m_{(i, \sigma, j ; t}(j)}$ outside of the locus of $\mathbb{F}_{i}$ given by $m_{\left(i, \sigma, j ; t^{(j)}\right)}=$ 0 . Our assumptions imply that this variety is not empty. Therefore, the polynomials of the equations above form a reduced regular sequence in $\mathbb{Q}[X, \mathbf{B}, \Lambda, \boldsymbol{\Theta}]_{m_{\left(i, \sigma, j ; t^{(j)}\right)}}$ and hence a lifting system in the sense of [23] for the variety $\mathcal{B}_{(i, \sigma, j)}$. Inductively we suppose that there is given a lifting fiber of $\mathcal{B}_{(i, \sigma, j)}$ on which $m_{\left(i, \sigma, j ; t^{(j)}\right)}$ vanishes nowhere.

In this situation $\Pi_{i}$ combines the algorithms "Lifting Curve", "Change Free Variables", "Change Lifting Point" and "Change Primitive Element" of [23] in order to produce a Kronecker-parameterization of a suitable curve $\mathcal{C}_{(i, \sigma, j)}$ in $\left(\mathcal{B}_{(i, \sigma, j)}\right)_{\left.m_{(i, \sigma, j, t}(j)\right)}$ which lifts the fiber of a sufficiently generic lifting point with respect to the lifting system and a sufficiently generic Noether position of $\mathcal{B}_{(i, \sigma, j)}$.

Next the procedure $\Pi_{i}$ applies for $1 \leq k \leq n-p-j+1$ the algorithm "One Dimensional Intersect" of 23 to the given Kronecker-parameterization of $\mathcal{C}_{(i, \sigma, j)}$ and the polynomials $M_{k}^{\left(i, \sigma, j-1, t^{(j-1)}\right)}$ and $m_{\left(i, \sigma, j-1, t^{(j-1)}\right)}$, and computes the greatest common divisor of the resulting univariate elimination polynomials. This greatest common divisor is not one since by assumption the variety $\mathcal{B}_{(i, \sigma, j-1)}$ is not empty. In this way $\Pi_{i}$ produces a lifting fiber of $\mathcal{B}_{(i, \sigma, j-1)}$ on which $m_{\left(i, \sigma, j-1, t^{(j-1)}\right)}$ vanishes nowhere.

Finally, $\Pi_{i}$ produces a geometric solution of the zero-dimensional algebraic variety $\mathcal{B}_{(i, \sigma, 1)}$. More precisely, the procedure $\Pi_{i}$ produces a circuit representation of the coefficients of $n(p+1)+1$ polynomials $P^{\sigma}, G_{1}^{\sigma}, \ldots, G_{n(p+1)}^{\sigma} \in \mathbb{Q}[Z]$ as above. 
Running the previous routine for each $\sigma_{k}, 1 \leq k \leq\left(\begin{array}{l}n \\ p\end{array}\right)$, we deduce from the complexity estimates of 23 ] that $\Pi_{i}$ uses

$$
\begin{gathered}
\sum_{1 \leq k \leq\left(\begin{array}{c}
n \\
p
\end{array}\right)} L(n d)^{O(1)}\left(\max \left\{\begin{array}{l}
\max \left\{\operatorname{deg}\left\{F_{1}=0, \ldots, F_{i}=0\right\} \mid 1 \leq i \leq p\right\}, \\
\max \left\{\operatorname{deg} S_{(r, l)}^{\left(i, \sigma_{k}\right)} \mid 1 \leq r \leq p, 1 \leq l \leq n\right\}, \\
\max \left\{\operatorname{deg} \mathcal{B}_{\left(i, \sigma_{k}, j\right)} \mid 1 \leq j \leq n-p\right\}
\end{array}\right\}\right)^{2} \\
=L(n d)^{O(1)}\left(\widetilde{\delta}_{i}\right)^{2}
\end{gathered}
$$

arithmetical operations organized, with respect to the parameters of the arithmetic circuit $\beta$, in non-scalar depth

$$
O\left(n(\ell+\log (d n)) \log \widetilde{\delta}_{i}\right) .
$$

The procedure can easily be translated to the bit model. Let $\eta$ be the logarithmic height of the polynomials $F_{1}, \ldots, F_{p}$. In order to estimate the bit complexity of the procedure we consider the maximal logarithmic height, say $\varkappa_{i}=O\left((n d)^{n} \eta\right)$, of the bipolar varieties $\mathcal{B}_{\left(i, \sigma_{k}, 1\right)}, 1 \leq k \leq\left(\begin{array}{l}n \\ p\end{array}\right)$. It is now straightforward to see that a representation of $P$ as primitive polynomial of $\mathbb{Z}[Z]$ and hence a minimal arithmetic expression of the real zeros of $P$ can be found using $O\left(L^{2}(n d \eta)^{O(1)}\left(\widetilde{\delta}_{i} \varkappa_{i}\right)^{2}\right)$ bit operations (see [25] for the relationship between arithmetic and bit representation of integers).

Let us finally observe that an alternative procedure to $\Pi_{i}$ may be obtained applying for $j:=1$ the algorithm "Geometric Solve " of [23] to the equation system of Proposition 7 The complexity estimates for this procedure, which are the same as for $\Pi_{i}$, follow from arguments in [5, Section 4] and especially from Theorem 3 and Example 2.

We have therefore proven the following complexity statement (compare [3, Theorem 11], 4, Theorem 13] and [8, Theorem 14].

Theorem 14. Let $n, p, d, i, \delta, L, \ell$ be natural numbers with $d \geq 1,1 \leq i \leq n-p$. Let $X_{1}, \ldots, X_{n}$ and $Z$ be indeterminates over $\mathbb{Q}$ and let $X:=\left(X_{1}, \ldots, X_{n}\right)$.

There exists an arithmetic network $\mathcal{N}$ (or arithmetic-Boolean circuit) over $\mathbb{Q}$, depending on certain parameters and having size

$$
O\left(L(n d)^{O(1)} \delta^{2}\right)=(n d)^{O(n)}
$$

and non-scalar depth

$$
O(n(\ell+\log (n d)) \log \delta)=O\left(n^{2} \log (d n) \log d\right),
$$

such that $\mathcal{N}$ satisfies for suitable random specializations of its parameters the following condition:

Let $F_{1}, \ldots, F_{p} \in \mathbb{Q}[X]$ be polynomials of degree at most $d$ and assume that $F_{1}, \ldots, F_{p}$ are given by an essentially division-free arithmetic circuit $\beta$ in $\mathbb{Q}[X]$ of size $L$ and non-scalar depth $\ell$. Suppose that $F_{1}, \ldots, F_{p}$ form a strongly reduced regular sequence in $\mathbb{Q}[X]$ and that $\widetilde{\delta}_{i} \leq \delta$ holds.

Then the algorithm represented by the arithmetic network $\mathcal{N}$ starts from the circuit $\beta$ as input and decides whether the variety $\left\{F_{1}=0, \ldots, F_{p}=0\right\}$ contains a smooth real point. If this is the case, the algorithm produces a circuit representation of the coefficients of $n+1$ polynomials $P, G_{1}, \ldots, G_{n} \in \mathbb{Q}[Z]$ satisfying for $G:=$ $\left(G_{1}, \ldots, G_{n}\right)$ the following conditions: 
- $P$ is monic and separable,

- $\operatorname{deg} G<\operatorname{deg} P \leq \delta$,

- the complex affine variety $\left\{G(z) \mid z \in \mathbb{A}^{1}, P(z)=0\right\}$ is zero-dimensional and contains a smooth real algebraic sample point for each generically smooth connected component of $\left\{F_{1}=0, \ldots, F_{p}=0\right\}_{\mathbb{R}}$.

In order to represent these sample points the algorithm returns an encoding " $\mathrm{a}$ la Thom" of the real zeros of the polynomial $P$.

For the terminology of arithmetic network and arithmetic Boolean circuit we refer to [46, 47].

Three remarks on the formulation of Theorem 14] are in order.

The statement of Theorem 14 remains true if we replace $\widetilde{\delta}_{i}$ by $\left(\begin{array}{l}n \\ p\end{array}\right) \delta_{i}$. This is a direct consequence of the estimate (11). Hence the combinatorial factor $\left(\begin{array}{l}n \\ p\end{array}\right)$ occurs hidden in the invariant $\widetilde{\delta}_{i}$. In terms of extrinsic complexity, our bounds therefore are comparable with those of [9]. An improvement can be obtained in the case that the variables $X_{1}, \ldots, X_{n}$ are in generic position with respect to the variety $\left\{F_{1}=\cdots=F_{p}=0\right\}$. In this case the factor $\left(\begin{array}{l}n \\ p\end{array}\right)$ in this complexity estimate may be dropped. In order to see this, observe that for the identity permutation $\sigma \in \operatorname{Sym}(n)$ the variety $H_{i, \sigma}$ contains for each generically smooth connected component $C$ of $\left\{F_{1}=\cdots=F_{p}=0\right\}_{\mathbb{R}}$ a point $(x, b, \lambda, \vartheta)$ such that $x$ belongs to $C$. Hence the same is true for $\mathcal{B}_{(i, \sigma, 1)}$. It suffices therefore to apply the subroutine of $\Pi_{i}$ which corresponds to $\sigma$ in order to find real algebraic sample points for the generically smooth connected components of $\left\{F_{1}=\cdots=F_{p}=0\right\}_{\mathbb{R}}$.

Next we remark that by (9) the condition $\widetilde{\delta}_{i} \leq \delta$ is always satisfied for $\delta:=$ $\min \left\{(n d)^{c n}, \delta_{i}\right\}$, where $c>0$ is a suitable universal constant (independent of $n$ and d). This illustrates that the estimates of Proposition 8 and Observation 11implying the estimate (8) (i.e., $\left.\delta_{i}=\left(n^{n} d\right)^{O(n)}\right)$ are not very relevant in this context. The worst case bound of Theorem 14 is only $(n d)^{O(n)}$.

Our third remark is the following statement.

Observation 15. Theorem 14 asserts only the existence of a computation that, for given $n$-variate input polynomials $F_{1}, \ldots, F_{p}$ of degree at most $d$ and circuit size and non-scalar depth $L$ and $\ell$, solves the real root finding problem for $F_{1}=$ $0, \ldots, F_{p}=0$ in sequential and non-scalar parallel time $O\left(L(n d)^{O(1)}\left(\widetilde{\delta}_{i}\right)^{2}\right)$ and $O\left(n(\ell+\log (n d)) \log \widetilde{\delta}_{i}\right)$, respectively.

Theorem 14 refers therefore to the non-uniform complexity model. In order to realize such a computation in terms of the uniform complexity model within the same order of sequential and parallel time, probabilistic methods have to be used (see [29] and [23]). This is achieved by choosing randomly the parameters of the arithmetic network $\mathcal{N}$ of Theorem 14

In [8, Section 5] we developed a common view for the procedures $\Pi_{i}, 1 \leq i \leq$ $n-p$, solving the task of finding smooth points in possibly singular, real compact hypersurfaces, and for the algorithm of [1] which solves the same task in the smooth case.

When we have to solve a concrete polynomial equation system $F_{1}=0, \ldots, F_{p}=$ 0 , sometimes the procedures $\Pi_{i}, 1 \leq i \leq n-p$ and algorithms of [2] may be combined in order to improve the complexity. However, such improvements depend on ad hoc methods and do not lead to a general algorithm. Moreover, 
the hypersurface case treated in [8] does not differ substantially from that of an arbitrary complete intersection. Therefore, we do not go into detail here and refer the reader to the above-mentioned paper.

\section{REFERENCES}

[1] B. Bank, M. Giusti, J. Heintz, and G. M. Mbakop, Polar varieties, real equation solving, and data structures: the hypersurface case, J. Complexity 13 (1997), no. 1, 5-27, DOI 10.1006/jcom.1997.0432. MR.1449757 (98h:68123)

[2] B. Bank, M. Giusti, J. Heintz, and G. M. Mbakop, Polar varieties and efficient real elimination, Math. Z. 238 (2001), no. 1, 115-144, DOI 10.1007/PL00004896. MR1860738 (2002g:14084)

[3] Bernd Bank, Marc Giusti, Joos Heintz, and Luis M. Pardo, Generalized polar varieties and an efficient real elimination procedure, Kybernetika (Prague) 40 (2004), no. 5, 519-550. MR2120995 (2006e:14078)

[4] B. Bank, M. Giusti, J. Heintz, and L. M. Pardo, Generalized polar varieties: geometry and algorithms, J. Complexity 21 (2005), no. 4, 377-412, DOI 10.1016/j.jco.2004.10.001. MR2152713 (2006f:14068)

[5] Bernd Bank, Marc Giusti, Joos Heintz, Mohab Safey El Din, and Eric Schost, On the geometry of polar varieties, Appl. Algebra Engrg. Comm. Comput. 21 (2010), no. 1, 33-83, DOI 10.1007/s00200-009-0117-1. MR2585564 (2011c:68065)

[6] Bernd Bank, Marc Giusti, Joos Heintz, and Luis Miguel Pardo, On the intrinsic complexity of point finding in real singular hypersurfaces, Inform. Process. Lett. 109 (2009), no. 19, 1141-1144, DOI 10.1016/j.ipl.2009.07.014. MR2552931(2010j:68036)

[7] Bernd Bank, Marc Giusti, Joos Heintz, and Luis Miguel Pardo, Bipolar varieties and real solving of a singular polynomial equation, Jaen J. Approx. 2 (2010), no. 1, 65-77. MR2789520 (2012e:14111)

[8] Bernd Bank, Marc Giusti, Joos Heintz, Lutz Lehmann, and Luis Miguel Pardo, Algorithms of intrinsic complexity for point searching in compact real singular hypersurfaces, Found. Comput. Math. 12 (2012), no. 1, 75-122, DOI 10.1007/s10208-011-9112-6. MR2886157

[9] Saugata Basu, Richard Pollack, and Marie-Françoise Roy, On the combinatorial and algebraic complexity of quantifier elimination, J. ACM 43 (1996), no. 6, 1002-1045, DOI 10.1145/235809.235813. MR1434910(98c:03077)

[10] Saugata Basu, Richard Pollack, and Marie-Françoise Roy, Algorithms in real algebraic geometry, 2nd ed., Algorithms and Computation in Mathematics, vol. 10, Springer-Verlag, Berlin, 2006. MR2248869 (2007b:14125)

[11] Peter Bürgisser, Michael Clausen, and M. Amin Shokrollahi, Algebraic complexity theory, Grundlehren der Mathematischen Wissenschaften [Fundamental Principles of Mathematical Sciences], vol. 315, Springer-Verlag, Berlin, 1997. With the collaboration of Thomas Lickteig. MR.1440179 (99c:68002)

[12] J.F. Canny, Some algebraic and geometric computations in PSPACE, ACM Symposium on Theory of Computing (STOC) (1988), 460-467.

[13] D. Castro, M. Giusti, J. Heintz, G. Matera, and L. M. Pardo, The hardness of polynomial equation solving, Found. Comput. Math. 3 (2003), no. 4, 347-420, DOI 10.1007/s10208-0020065-7. MR2009683 (2004k:68056)

[14] M. Coste and M.-F. Roy, Thom's lemma, the coding of real algebraic numbers and the computation of the topology of semi-algebraic sets, J. Symbolic Comput. 5 (1988), no. 1-2, 121-129, DOI 10.1016/S0747-7171(88)80008-7. MR949115 (89g:12002)

[15] C. D' Andrea, T. Krick, and M. Sombra, Heights of varieties in multi-projective spaces and arithmetic Nullstellensätze, Manuscript, Universidad de Buenos Aires (2011)

[16] M. Demazure, Catastrophes et bifurcations, Ellipses, Paris 1989.

[17] Clémence Durvye and Grégoire Lecerf, A concise proof of the Kronecker polynomial system solver from scratch, Expo. Math. 26 (2008), no. 2, 101-139, DOI 10.1016/j.exmath.2007.07.001. MR2413831 (2009c:14119)

[18] William Fulton, Intersection theory, 2nd ed., Ergebnisse der Mathematik und ihrer Grenzgebiete. 3. Folge. A Series of Modern Surveys in Mathematics [Results in Mathematics and Related Areas. 3rd Series. A Series of Modern Surveys in Mathematics], vol. 2, SpringerVerlag, Berlin, 1998. MR 1644323 (99d:14003) 
[19] M. Giusti, J. Heintz, J. E. Morais, and L. M. Pardo, When polynomial equation systems can be "solved" fast?, (Paris, 1995), Lecture Notes in Comput. Sci., vol. 948, Springer, Berlin, 1995, pp. 205-231, DOI 10.1007/3-540-60114-7_16. MR1448166 (98a:68106)

[20] M. Giusti, J. Heintz, K. Hägele, J. E. Morais, L. M. Pardo, and J. L. Montaña, Lower bounds for Diophantine approximations, J. Pure Appl. Algebra 117/118 (1997), 277-317, DOI 10.1016/S0022-4049(97)00015-7. Algorithms for algebra (Eindhoven, 1996). MR.1457843 (99d:68106)

[21] M. Giusti, J. Heintz, J. E. Morais, J. Morgenstern, and L. M. Pardo, Straight-line programs in geometric elimination theory, J. Pure Appl. Algebra 124 (1998), no. 1-3, 101-146, DOI 10.1016/S0022-4049(96)00099-0. MR 1600277 (99d:68128)

[22] Marc Giusti and Joos Heintz, Kronecker's smart, little black boxes, Foundations of computational mathematics (Oxford, 1999), London Math. Soc. Lecture Note Ser., vol. 284, Cambridge Univ. Press, Cambridge, 2001, pp. 69-104. MR.1836615 (2002e:65075)

[23] Marc Giusti, Grégoire Lecerf, and Bruno Salvy, A Gröbner free alternative for polynomial system solving, J. Complexity 17 (2001), no. 1, 154-211, DOI 10.1006/jcom.2000.0571. MR:1817612(2002b:68123)

[24] D. Yu. Grigor'ev and N. N. Vorobjov Jr., Solving systems of polynomial inequalities in subexponential time, J. Symbolic Comput. 5 (1988), no. 1-2, 37-64, DOI 10.1016/S07477171(88)80005-1. MR 949112(89h:13001)

[25] K. Hägele and J. L. Montaña, Polynomial random test for the equivalence of integers given by arithmetic circuits, Depto. de Matematicas, Estadistica y Computacion, Universidad de Cantabria, 4 (1997)

[26] Joos Heintz, Definability and fast quantifier elimination in algebraically closed fields, Theoret. Comput. Sci. 24 (1983), no. 3, 239-277, DOI 10.1016/0304-3975(83)90002-6. MR716823 (85a:68062)

[27] Joos Heintz, Bart Kuijpers, and Andrés Rojas Paredes, Software Engineering and complexity in effective algebraic geometry, J. Complexity 29 (2013), no. 1, 92-138, DOI 10.1016/j.jco.2012.04.005. MR2997853

[28] J. Heintz, B. Kuijpers, A. Rojas Paredes, On the intrinsic complexity of elimination problems in effective algebraic geometry, arXiv:1201.4344v3.

[29] Joos Heintz, Guillermo Matera, and Ariel Waissbein, On the time-space complexity of geometric elimination procedures, Appl. Algebra Engrg. Comm. Comput. 11 (2001), no. 4, 239-296, DOI 10.1007/s002000000046. MR1818975(2002c:68108)

[30] J. Heintz, M.-F. Roy, and P. Solernó, On the complexity of semialgebraic sets, in IFIP Information Processing 89 (G. X. Ritter, ed.), Elsevier, 1989, pp. 293-298.

[31] Joos Heintz, Marie-Françoise Roy, and Pablo Solernó, Complexité du principe de TarskiSeidenberg, C. R. Acad. Sci. Paris Sér. I Math. 309 (1989), no. 13, 825-830 (French, with English summary). MR1055203 (92c:12012)

[32] Joos Heintz, Marie-Françoise Roy, and Pablo Solernó, Sur la complexité du principe de TarskiSeidenberg, Bull. Soc. Math. France 118 (1990), no. 1, 101-126 (French, with English summary). MR,1077090(92g:03047)

[33] George Kempf, On the geometry of a theorem of Riemann, Ann. of Math. (2) 98 (1973), 178-185. MR0349687 (50 \#2180)

[34] Alexander Morgan and Andrew Sommese, A homotopy for solving general polynomial systems that respects m-homogeneous structures, Appl. Math. Comput. 24 (1987), no. 2, 101-113, DOI 10.1016/0096-3003(87)90063-4. MR914806 (88j:65110)

[35] J. Renegar, A faster PSPACE algorithm for the existential theory of the reals, in Proc. 29th Annual IEEE Symposium on the Foundation of Computer Science, 1988, pp. 291-295.

[36] James Renegar, On the computational complexity and geometry of the first-order theory of the reals. I. Introduction. Preliminaries. The geometry of semi-algebraic sets. The decision problem for the existential theory of the reals, J. Symbolic Comput. 13 (1992), no. 3, 255-299, DOI 10.1016/S0747-7171(10)80003-3. MR.1156882 (93h:03011a)

[37] T. G. Room, The geometry of determinantal loci, Cambridge Univ. Press (1938).

[38] F. Severi, Sulle intersezioni delle varieta algebriche e sopra $i$ loro caratteri e singolarita proiettive, Torino Mem. (2) 52 (1903), 61-118.

[39] Francesco di Severi, La serie canonica e la teoria delle serie principali di gruppi di punti sopra una superficie algebrica, Comment. Math. Helv. 4 (1932), no. 1, 268-326, DOI 10.1007/BF01202721 (Italian). MR1509461 
[40] Michael Spivak, Calculus on manifolds. A modern approach to classical theorems of advanced calculus, W. A. Benjamin, Inc., New York-Amsterdam, 1965. MR0209411 (35 \#309)

[41] Bernard Teissier, Variétés polaires. II. Multiplicités polaires, sections planes, et conditions de Whitney, Algebraic geometry (La Rábida, 1981), Lecture Notes in Math., vol. 961, Springer, Berlin, 1982, pp. 314-491, DOI 10.1007/BFb0071291 (French). MR708342 (85i:32019)

[42] Bernard Teissier, Quelques points de l'histoire des variétés polaires, de Poncelet à nos jours, Séminaire d'Analyse, 1987-1988 (Clermont-Ferrand, 1987), Univ. Clermont-Ferrand II, Clermont, 1990, pp. Exp. No. 4, 12 (French). MR1088966 (91m:14001)

[43] J. A. Todd, The Geometrical Invariants of Algebraic Loci, Proc. London Math. Soc. S2-43, no. 2, 127, DOI 10.1112/plms/s2-43.2.127. MR1575589

[44] J. A. Todd, The Arithmetical Invariants of Algebraic Loci, Proc. London Math. Soc. S2-43, no. 3, 190, DOI 10.1112/plms/s2-43.3.190. MR1575915

[45] W. Vogel, Lectures on results on Bezout's theorem, Tata Institute of Fundamental Research Lectures on Mathematics and Physics, vol. 74, Published for the Tata Institute of Fundamental Research, Bombay, 1984. Notes by D. P. Patil. MR743265 (86f:14003)

[46] Joachim von zur Gathen, Parallel arithmetic computations: a survey, Mathematical foundations of computer science, 1986 (Bratislava, 1986), Lecture Notes in Comput. Sci., vol. 233, Springer, Berlin, 1986, pp. 93-112, DOI 10.1007/BFb0016236. MR874591

[47] J. von zur Gathen, Parallel linear algebra, in Synthesis of parallel algorithms (J. H. Reif, ed.), Kaufmann, San Mateo, CA., 1993, pp. 573-617.

Institut für Mathematik, Humboldt-Universität zu Berlin, Unter den Linden 6, D10099 Berlin, Germany

E-mail address: bank@mathematik.hu-berlin.de

CNRS, École Polytechnique, Lab. LiX, F-91228 Palaiseau, Cedex, France

E-mail address: marc.giusti@polytechnique.fr

Departamento de Computación, Universidad de Buenos Aires, COniCET, Ciudad Universitaria, Pabellon I, 1428 Buenos Aires, Argentina - Departamento de Matemáticas, Estadística y Computación, Facultad de Ciencias, Universidad de Cantabria, Avda. de los Castros, E-39005 SAntander, Spain

E-mail address: joos@dc.uba.ar 